\title{
TRABAJOS DE PROTECCIÓN EN LOS SITIOS ARQUEOLÓGICOS CONSIDERADOS DENTRO DE LAS ACCIONES DE PREVENCIÓN POR EL FENÓMENO EL NIÑO 2015 EN LA REGIÓN LAMBAYEQUE ${ }^{* *}$
}

\author{
CONSIDERED PROTECTION MEASURES AT ARCHAEOLOGICAL SITES CONSIDERED WITHIN \\ THE PREVENTIVE ACTIONS AGAINST THE 2015 EL NIÑO PHENOMENON \\ IN THE LAMBAYEQUE REGION
}

\author{
ALBERTO COAHILA OSORIO(**) \\ Fecha de recepción: 16 abril de 2016 \\ Fecha de aprobación: 28 de mayo de 2016
}

\begin{abstract}
RESUMEN
La región Lambayeque posee un rico legado cultural, cuyas evidencias, accesibles mediante investigaciones arqueológicas, colaboran con la construcción de nuestra identidad y a la generación de conocimiento. Por lo tanto, es importante proteger esta evidencia arqueológica y asegurar su perdurabilidad para el disfrute de futuras generaciones. El Gobierno central, a través del Ministerio de Cultura, emprendió un programa de protección del patrimonio arqueológico en la costa norte del país con la finalidad de mitigar el impacto de las lluvias del fenómeno de El Niño (ENSO - El Niño Southern OsciIlation). Estas actividades en la región Lambayeque fueron canalizadas a través de la Unidad Ejecutora 005 Naylamp entre finales de 2014 e inicios de 2016, y abarcaron 28 de los sitios arqueológicos más vulnerables. Las acciones de conservación ejecutadas permitieron preparar a las áreas de intervención y dejarlas en las mejores condiciones posibles para poder enfrentar los embates de la naturaleza con mayor éxito. El siguiente artículo da a conocer sobre la territorialidad del patrimonio arqueológico en la región Lambayeque, según el diagnóstico físico espacial elaborado en el año 2015 como parte del Plan Maestro para el Mejor Manejo del Patrimonio Arqueológico de la Región Lambayeque.
\end{abstract}

\section{PALABRAS CLAVE}

Fenómeno de El Niño, prevención, patrimonio arqueológico de la Región Lambayeque

\begin{abstract}
The Lambayeque region, which possesses a rich cultural heritage, contains archaeological evidence that helps build our identity; this can occur thanks to the presence of societies that evolved and put forth the knowledge they obtained through research. Therefore, it is important to protect such archeological evidence and ensure its durability for the take profit of future generations. The national government, through the Ministry of Culture, launched a program for the protection of archaeological heritage in the northern coast of the country in order to mitigate the impact of rains of El Niño (ENSO -El Niño Southern Oscillation). Activities in the Lambayeque region were channeled through the Executive Unit 005 Naylamp from late 2014 until early 2016, and covered a total of 28 of the most vulnerable archaeological sites. The preservation procedures carried out allowed the selected areas to prepared in the best conditions and with better results against ravages of nature. Finally, we reflect about the territoriality of archaeological heritage in the Lambayeque region, according to the spatial-physical diagnosis developed in 2015 as part of the Master Plan for a Better Management of Archaeological Heritage of the Region Lambayeque.
\end{abstract}

\section{KEYWORDS}

El Niño phenomenon, prevention, archaeological heritage of Lambayeque Region

(*) El presente artículo se desarrolla a partir del análisis de los informes de evaluación elaborados por la Unidad Ejecutora 005 Naylamp Lambayeque sobre los trabajos de prevención realizados en los sitios arqueológicos de la región Lambayeque, entre octubre de 2014 y enero de 2016, con respecto a la presencia del Fenómeno de El Niño en esta parte del país. Esta evaluación se complementa con el resumen del diagnóstico físico-espacial (territorial) del Plan Maestro para los Sitios Arqueológicos de la Región Lambayeque, que se viene elaborando desde 2015.

(**) Arquitecto, graduado de la Universidad Nacional San Antonio Abad el Cusco, con estudios de maestría en Conservación del Patrimonio Edificado (Universidad Nacional de Ingeniería) y experiencia profesional especializada en patrimonio cultural como arquitecto responsable del Área de Conservación del Complejo Arqueológico Chankillo (2012-2013), responsable del Área de Conservación y Restauración en el Proyecto de Puesta en Valor de la Zona Arqueológica Monumental de Huaycán de Pariachi (2013-2014), y responsable de estudios y proyectos de la Unidad de Infraestructura y Proyectos de la Unidad Ejecutora 005 Naylamp Lambayeque (abril 2014 - actualidad). Contacto: betocoa@hotmail.com 
En el siglo XIX, según señalan algunas fuentes, los pescadores del norte del Perú fueron los primeros en identificar el fenómeno de El Niño, descrito como una corriente de aguas cálidas con rumbo de norte a sur, que afecta a Perú y Ecuador, y cuya presencia se da en el mes de diciembre, por lo que se le otorgó esta denominación en alusión a la Navidad. Desde ese momento se inició un interés de carácter científico sobre su impacto, a raíz de un fenómeno de tropicalización que se extiende hasta Pisco, en el sur, como señala Morales:

Desde entonces se define este fenómeno como una contracorriente de aguas cálidas que aparecía a inicios del verano con impactos en la biomasa. Posteriormente se plantean las primeras cuestiones sobre una posible correspondencia de esta corriente marina con las intensas Iluvias que afectaban las cotas ecuatoriana y peruana. Desde entonces se reconoce El Niño como un fenómeno a nivel regional y décadas después se califica como un evento de carácter global. (2014, p. 77)

En la pasada centuria la frecuencia histórica de los ENSO registra eventos fuertes "1925, 1982, 1997", siendo catastróficos, pero de diferente comportamiento, de los cuales no se encuentra un registro instrumental ni una evaluación comparativa confiable en las diferentes regiones donde tiene incidencia este fenómeno, teniendo como indicios algunos factores denominados bio indicadores [sic] que denotan un comportamiento atípico de floraciones prematuras, cambios de coloración del celaje o la alteración de la biomasa de especies terrestres como acuáticas, factores que sirven de alerta sobre la amenaza y cambiós [sic] inmediatos. $(2014$, p. 1$)$

A partir de las dos experiencias pasadas, se puede entender el grado de destrucción que genera este fenómeno climático en Tumbes, Piura, Lambayeque y la Libertad. Este se expresa en diferentes magnitudes, desde leve y moderado hasta fuerte, pero es una tragedia cada vez que se manifiesta, situación agravada por la escasa o casi nula política preventiva del Gobierno respecto del manejo de las cuencas y microcuencas. Este fenómeno climático provoca daños al patrimonio arqueológico, pues la presencia de inundaciones y desbordes ha alterado las estructuras arqueológicas que aún se mantienen en pie, y continúan a merced de sufrir más daños, especialmente si persiste la inexistencia de acciones preventivas de monitoreo y control. Estas no son planificadas con anticipación a pesar de la inminencia del fenómeno, característico de la época del año señalada.

La experiencia vivida durante manifestaciones pasadas del fenómeno de El Niño en la región Lambayeque demuestra que, al no existir planes de contingencia a nivel de los diversos sectores (gobiernos locales y regionales), este ha tenido un fuerte impacto en el grado de conservación del patrimonio cultural edificado de la misma. Por eso es importante tener una articulación entre todos los actores involucrados, como los gobiernos locales y regionales, los cuales deben trabajar de manera conjunta durante las diversas fases (mitigación, preparación y respuesta), así como que se cumplan los roles asignados a la población e instituciones a cargo de la organización. De acuerdo a este planteamiento, se están tomando las medidas a través de planes de prevención y la programación de acciones de contingencia, a fin de estar preparados ante la ocurrencia de escenarios no deseados. En ese sentido, las acciones de prevención y contingencia deben desarrollar trabajos tanto de mitigación como de respuesta inmediata, que gestionen la forma cómo actuar una vez que se activen los escenarios de riesgo.

La región Lambayeque, poseedora de un cúmulo de valores históricos, únicos en el Perú por su ubicación en la costa norte, configura un paisaje con fuerte acervo cultural edificado, entre construcciones prehispánicas e históricas, las cuales se encuentran en un alto riesgo de afectación. Asimismo, debido al emplazamiento de los sitios arqueológicos, estos están categorizados dentro de la cadena de riesgo como un grupo potencial de alto grado de deterioro frente a eventos climático de la magnitud de un fenómeno de El Niño (Morales, 2014). 
Debido a esto, la Unidad Ejecutora 005 Naylamp programó acciones de prevención ante el fenómeno de El Niño en el año 2015, en los sitios arqueológicos que se encuentran actualmente en investigación y los que están abiertos al público, con la finalidad de mitigar su impacto.

\section{Antecedentes}

Los reportes brindados el año 2014, en los meses de julio a marzo, sobre la formación de un ENSO con proyección de leve a moderado, generaron una alerta para la costa norte del país y en particular para el sector cultura, teniendo en cuenta los eventos anteriores y el grado de destrucción que estos ocasionaron sobre el patrimonio cultural, principalmente en tierra, en 1983 y 1998.

Señala Morales que "la realidad del patrimonio arqueológico en el Perú presenta una problemática diversa, compleja y complicada. Debido a la natural fragilidad de los materiales constructivos y la fatiga físico-mecánica de estos" (2014, p. 1). A este factor se suma la vulnerabilidad generada por las condiciones de los eventos naturales, como se menciona en el párrafo anterior. En este contexto, y ante el pronóstico de eventos climáticos adversos, el Ministerio de Cultura inició una política de manejo de esta amenaza a través de sus órganos descentralizados, mediante acciones de prevención, y planes de contingencia para los sitios arqueológicos e históricos y museos de la región Lambayeque. Esta política tuvo como primer objetivo proteger a las estructuras arqueológicas de las lluvias estacionarias, principalmente durante los meses de diciembre a marzo, con miras a un fenómeno ENSO de intensidad fuerte, como el que fue anunciado para el año 2015. Bajo este contexto se empezaron a formular y trazar objetivos siguiendo la premisa de la prevención, por lo que se iniciaron trabajos de evaluación de las áreas a intervenir, a través de un registro y evaluación exhaustivos. Esto resultó útil en la medida en que se reunió información para poder dar respuesta a cuestiones básicas, a fin de poder implementar un plan de prevención, pues se identificaron, dentro del vasto número de sitios a intervenir, los más vulnerables frente a las variables de clasificación que se plantearon durante este proceso.

\section{Descripción de las actividades}

Partiendo de la premisa de la prevención, se planificaron acciones de conservación a través de la elaboración de una metodología con actividades para las etapas de mitigación, prevención y resiliencia, en las que se incluyeron acciones de corto, mediano y largo plazo, orientadas a paliar los daños que los diversos factores causan a las estructuras arqueológicas, así como para protegerlas durante y después del evento. En ese sentido, señala Morales lo siguiente:

...se plantearon objetivos con la finalidad de obtener la caracterización del estado actual de las condiciones ambientales y físicas que inciden sobre el patrimonio con la finalidad de proponer un plan de contingencia a través de una sectorización programando acciones para mitigar el impacto de un fenómeno climático sobre el patrimonio edificado, fomentar el intercambio de experiencias profesionales en temas de conservación del patrimonio cultural consensuando principios, criterios, técnicas y materiales en los tratamientos. (2014, p. 45)

También se buscó promover la participación activa de las poblaciones adyacentes en las actividades de protección de los monumentos (ver Figura 1).

La sistematización de esta etapa facilitó conocer las características de los sitios a proteger, así como su grado de deterioro y las posibles causas que agravarían tal estado. Así mismo, permitió plantear las acciones a realizar en cada una de las áreas de intervención. Este análisis de datos basado en las estimaciones de campo permitió reconocer cómo serían los escenarios de afectación en cada sector o elemento. Para ello, se empezó por establecer una primera cuantificación de los daños, y se llegó a presupuestar 


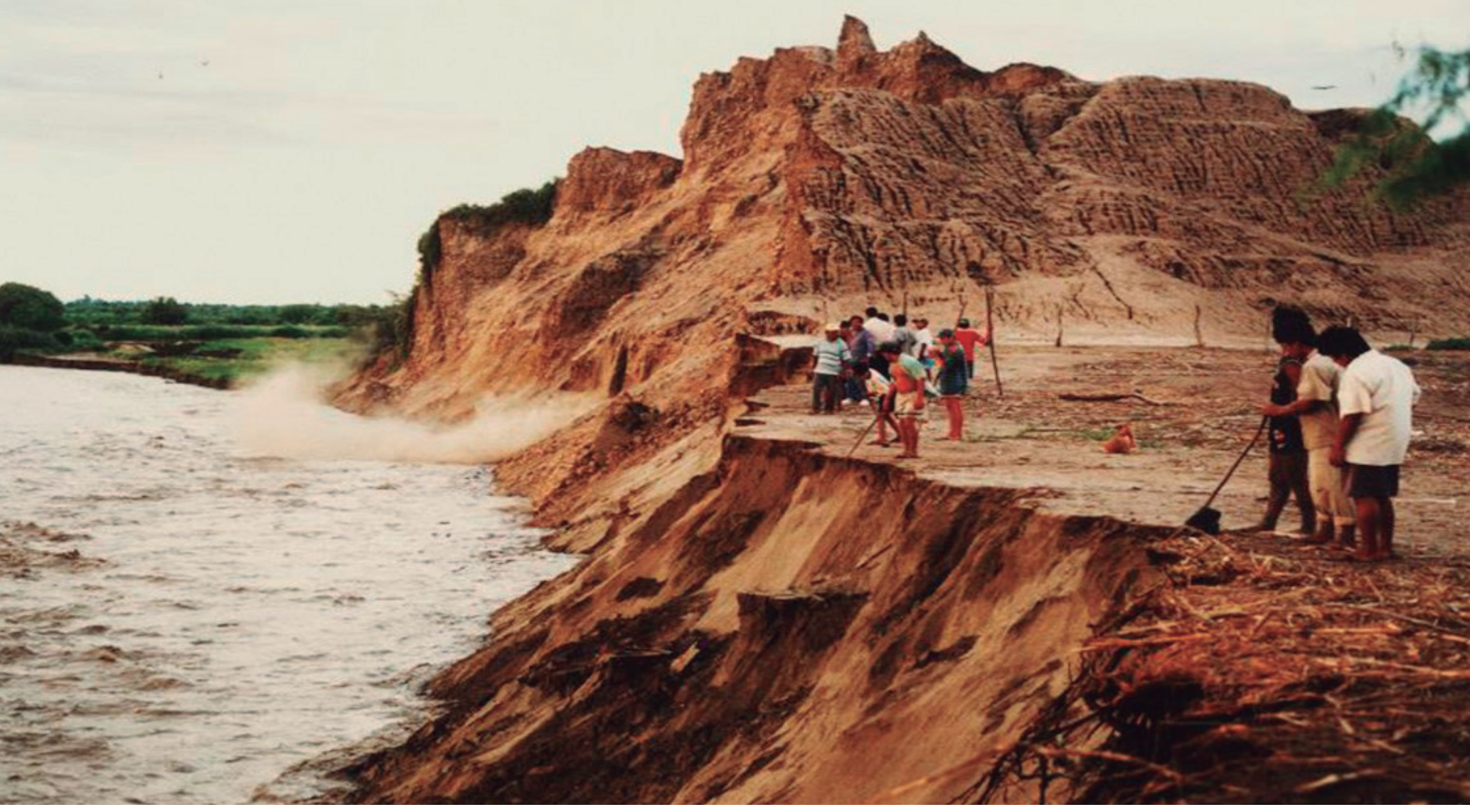

las acciones de emergencia adecuadas para cada caso, detallando los principales componentes. Esta información fue sistematizada en fichas técnicas, las cuales contenían la síntesis cualitativa y cuantitativa de las áreas a intervenir. (Ver Tabla 1)

Los trabajos de prevención estuvieron orientados a realizar acciones mediante la ejecución de trabajos de protección de estructuras, como la instalación de coberturas temporales, levantadas con estructura de caña Guayaquil y planchas de fibra vegetal; sistemas de evacuación de aguas y defensas ribereñas; drenajes subterráneos y suspendidos; limpieza de cursos de agua; entre otras acciones de conservación directa, como la instalación de muros de contacto para la protección de las estructuras arqueológicas. Todas estas acciones tuvieron la finalidad de mitigar el daño que pudiera ocasionar la presencia de factores climáticos, principalmente los producidos por las lluvias estacionarias, y eventos de mayor magnitud, como el anunciado para el año 2015. (Ver Tabla 2)

\section{Primera etapa}

Dentro de la primera etapa, las actividades a desarrollar fueron programadas a través de la elaboración de una ficha técnica que sirvió como documento guía para el proceso de intervención, a nivel técnico y presupuestal. En este documento se sistematizaron las actividades de acuerdo a las evaluaciones realizadas con anterioridad, lo cual se tradujo en un diagnóstico de cada una de las áreas de intervención. Esto permitió tener una mejor aproximación a la realidad, y así poder plantear las diversas acciones y mecanismo de intervención.

Los trabajos a desarrollar consistieron en la construcción de coberturas, como principal acción que fue complementada por otras como la instalación de drenajes suspendidos y subterráneos, y de defensas ribereñas; el desbroce y la limpieza de cursos de agua; y el tratamiento de quebradas. Asimismo, se abordaron actividades de conservación directa (colocación de muros de contacto, apuntalamientos e instalación de pisos de sacrifico, entre las más importantes) (ver Figuras 2 y 3 ).
Figura 1. La activación y crecida de los cursos de agua están consideradas dentro de los factores que mayor deterioro causan al patrimonio arqueológico.

Fuente: Wester, 1998, en Morales, 2014 
devenir Vol. 3, N5, ENERO - JUNIO 2016, PP. 90-112 - ESTUDIOS I ISSN 2312-7570

UnIVERSIDAD NACIONAL DE INGENIERÍA, LIMA

Tabla 1. Descripción de las etapas de prevención por el fenómeno de El Niño realizadas en el año 2015

\begin{tabular}{|c|c|c|}
\hline & Actividad & Metas programadas \\
\hline \multirow{7}{*}{ Primera etapa } & \multirow{7}{*}{ Actividades de prevención } & Instalación de coberturas temporales \\
\hline & & Reforzamiento de riberas \\
\hline & & Limpieza y desbroce de cursos de agua \\
\hline & & Instalación de drenajes suspendidos \\
\hline & & Instalación de drenajes subterráneos \\
\hline & & Sellado de pozos de huaqueo \\
\hline & & Instalación de cortavientos \\
\hline \multirow{2}{*}{ Segunda etapa } & \multirow{2}{*}{ Plan de contingencia } & Ampliación de coberturas \\
\hline & & Trabajos complementario en drenajes suspendidos y subterráneos \\
\hline
\end{tabular}

Fuente: Elaboración Alberto Coahila, 2015.

Tabla 2. Clasificación de las áreas de intervención de la primera etapa

\begin{tabular}{|c|c|c|c|}
\hline \multicolumn{2}{|c|}{ Ubicación } & \multirow{2}{*}{ Código } & \multirow{2}{*}{ Áreas de intervención } \\
\hline Provincia & Distrito & & \\
\hline \multirow{14}{*}{ Chiclayo } & \multirow{3}{*}{ Patapo } & L 1 & Sitio Arqueológico Huaca Brava \\
\hline & & L 2 & Sitio Arqueológico Cerro Patapo \\
\hline & & L 2.1 & Sitio Arqueológico El Tambo \\
\hline & Tumán & L 8 & Sitio Arqueológico Cerro Luya \\
\hline & \multirow{2}{*}{ Zaña } & L 3 & Sitio Arqueológico Huaca Rajada - Sipán \\
\hline & & L 4 & Sitio Arqueológico Saltur \\
\hline & Chongoyape & L5 & Complejo Arqueológico Pampa Grande \\
\hline & \multirow{2}{*}{ Pucalá } & L 6 & Sitio Arqueológico La Inmaculada \\
\hline & & L 7 & Sitio Arqueológico Huaca Santa Rosa \\
\hline & \multirow{2}{*}{ Pomalca } & L 21 & Sitio Arqueológico Ventarrón \\
\hline & & L 22 & Complejo Arqueológico Collud Zarpán \\
\hline & Lagunas & L 10 & Complejo Murales de Ucupe \\
\hline & Tumán & L 24 & Templo Colonial San Juan de la Punta \\
\hline & Zaña & L 25 & Complejo Arquitectónico Religioso Zaña \\
\hline \multirow{10}{*}{ Lambayeque } & \multirow{3}{*}{ Túcume } & L 23 & Templo Colonial Túcume Viejo \\
\hline & & L 14 & Complejo Arqueológico Túcume - Huaca Larga \\
\hline & & L 15 & Sitio Arqueológico Huaca Manuelón \\
\hline & \multirow{2}{*}{ Lambayeque } & L 17 & Complejo Arqueológico Chotuna \\
\hline & & L 18 & Complejo Arqueológico Chornancap \\
\hline & Jayanca & L 19 & Sitio Arqueológico Cerro Jotoro \\
\hline & Lambayeque & L 20 & Sitio Arqueológico Huaca Los Perros \\
\hline & Mochumi & L 20.1 & Sitio Arqueológico Huaca La Pava \\
\hline & \multirow{2}{*}{ Pacora } & \multirow{2}{*}{ L 26} & Zona Arqueológica Monumental Huaca Bandera - CA1 \\
\hline & & & Zona Arqueológica Monumental Huaca Bandera - H. Blanca \\
\hline \multirow{2}{*}{ Ferreñafe } & \multirow{2}{*}{ Ptipo } & L 11 & Sitio Arqueológico Huaca Las Ventanas \\
\hline & & $\mathrm{L} 12$ & Sitio Arqueológico Huaca Lercarlech \\
\hline \multirow{2}{*}{ Lambayeque } & \multirow{2}{*}{ Lambayeque } & L9 & Museo Tumbas Reales de Sipán \\
\hline & & L 16 & Museo Bruning \\
\hline Ferreñafe & Ferreñafe & L 13 & Museo Sicán \\
\hline
\end{tabular}




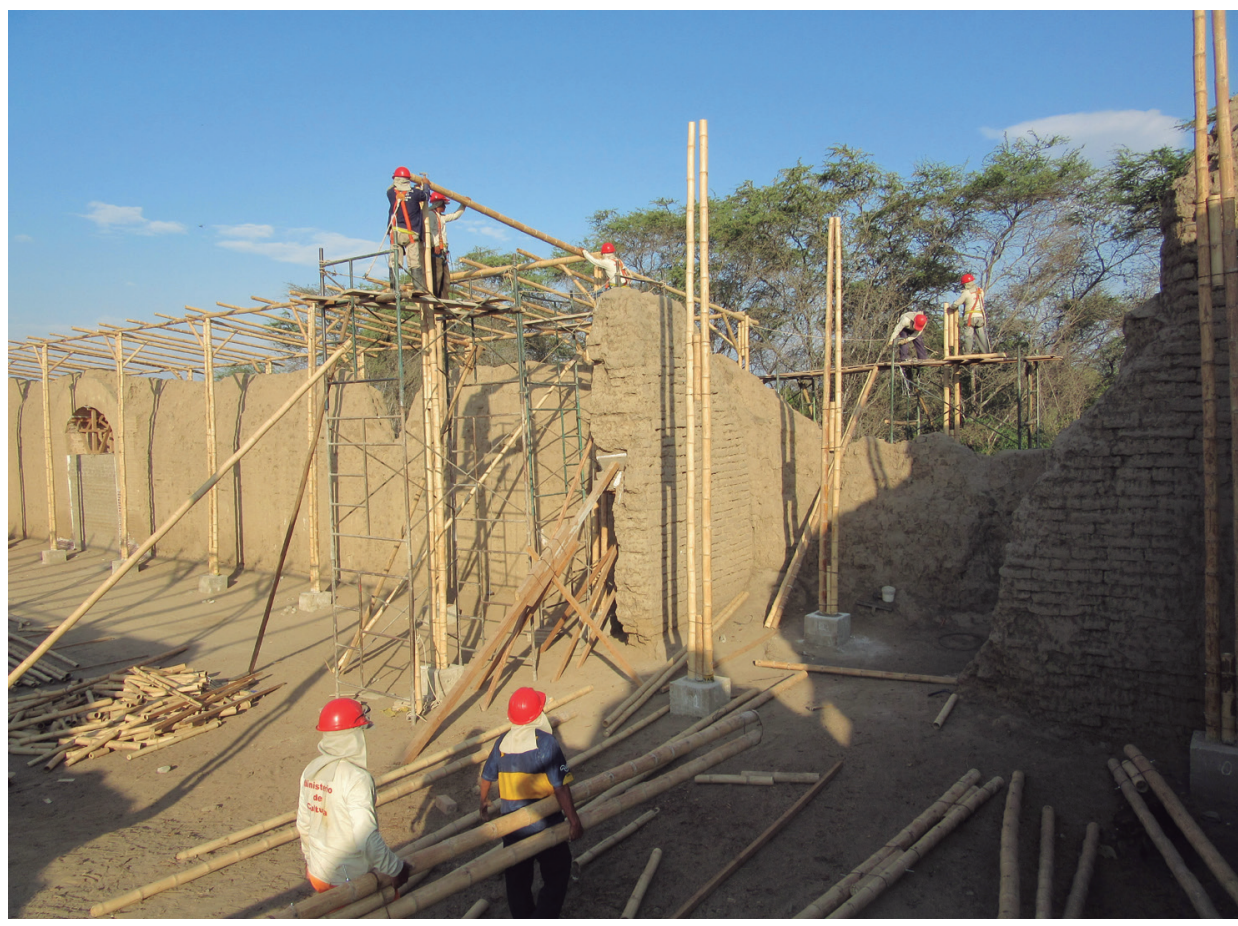

Figura 2. Templo Colonial Túcume Viejo: instalación de coberturas sobre los muros que aún se mantienen en pie. Nótese el uso de caña Guayaquil como estructura de soporte.

Fuente: Archivo fotográfico personal Alberto Coahila, 2014

Figura 3. Huaca las Ventanas - Bosque de Pomac: instalación de sistemas de evacuación de aguas pluviales conformados por tubos de PVC de 6"

Fuente: Archivo fotográfico personal Alberto Coahila, 2014.

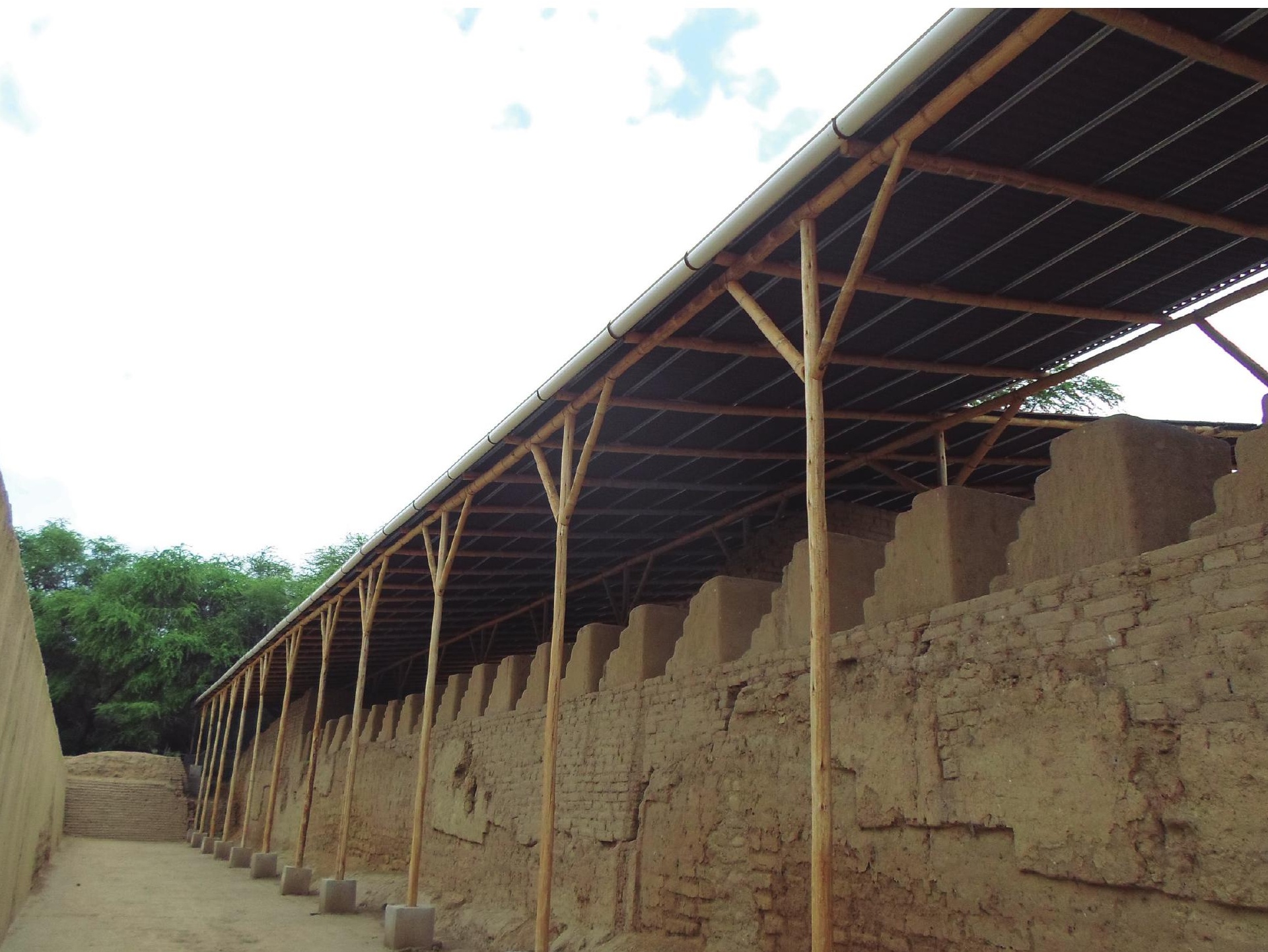


devenir Vol. 3, N5, ENERO - JUNIO 2016, PP. 90-112 - EsTUDIOS I ISSN 2312-7570

UNIVERSIDAD NACIONAL DE INGENIERÍ, LIMA

Figura 4. Montaje de estructuras para la cobertura. Instalación de muretes con sacos de arena para impedir el ingreso de agua

Fuente: Archivo fotográfico personal Alberto Coahila, 2014.

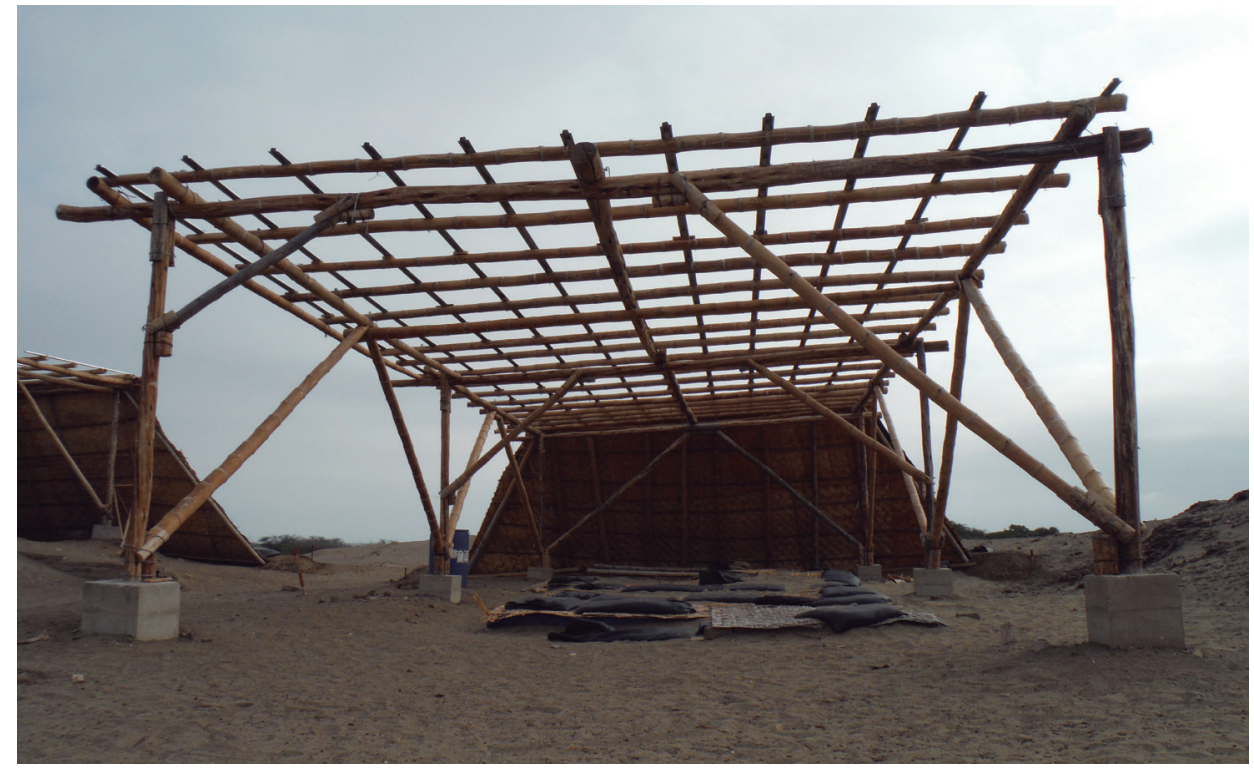

\section{a. Descripción de las acciones}

Respecto a la instalación de las coberturas, el principal trabajo consistió en el montaje de nuevas coberturas temporales. En las labores de montaje de la nueva cubierta para cada sitio intervenido, el trabajo desarrollado se realizó siguiendo los mismos principios que las estructuras preexistentes (soporte de madera rollizo y caña Guayaquil), con coberturas de fibra vegetal, con la finalidad de proteger las áreas excavadas, que se encontraban expuestas. Asimismo, se procedió a proteger las estructuras que aún quedan en pie y algunas zonas de coberturas de los museos existentes de la región.

La instalación de dichas cubiertas fue respaldada mediante las charlas técnicas brindadas en cada sitio de intervención sobre la forma y técnica que debe utilizarse para su instalación. Finalmente, los criterios de ejecución para las instalaciones de todas las coberturas siguieron los mismos principios de lo ya instalado. Esto implica una uniformidad en el planteamiento para levantar el sistema integral de montaje (Coahila, 2016).

En cuanto a la evacuación de aguas pluviales, los trabajos estuvieron dirigidos a la instalación de drenajes a nivel de piso y en altura, con la finalidad de recibir el caudal de emisión que captan las coberturas en eventos de lluvia.

Como parte de las acciones de reforzamiento o defensa, se realizaron trabajos de instalación de muros de contención en la margen de ríos y pequeños cauces de agua secos. En algunos casos se utilizó sacos de arena, y en otros se levantaron muros en seco o en piedra, con la finalidad de poder controlar el impacto de los flujos de agua que discurren al producirse eventos lluviosos, para evitar que generen erosión y pongan en peligro las estructuras arqueológicas que se encuentran aún en pie.

Como acción de conservación directa, se levantaron muros de contacto para proteger y estabilizar muros con áreas inestables. También, se realizaron trabajos de apuntalamiento de muros que presentaban fallas estructurales, los cuales se encontraban en avanzado estado de deterioro, así como de protección de pisos originales con superficies de sacrificio (ver Figuras 4 y 5 ). 


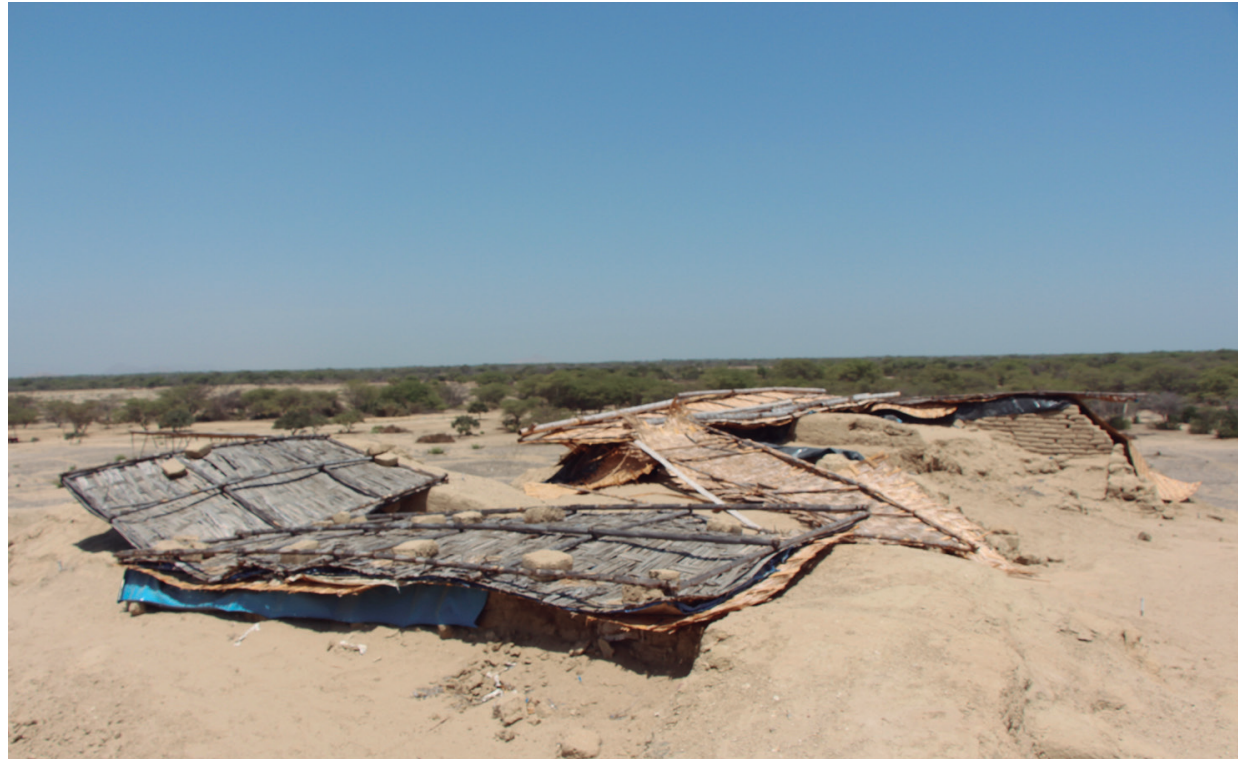

Acciones complementarias fueron los trabajos de limpieza de las áreas por donde discurre el agua producida por las lluvias, a fin de evitar una acumulación de la misma y la formación de pozos en áreas de relieve plano.

\section{Segunda etapa}

Como trabajos complementarios, entre de las acciones de prevención, se programó una segunda etapa, denominada 'trabajos de contingencia', con la finalidad de realizar acciones complementarias de protección. Las áreas de intervención fueron elegidas debido a su vulnerabilidad y fragilidad frente al impacto de los agentes atmosféricos: se privilegió aquellas que corrían grave riesgo de ser afectadas por su ubicación en laderas o por el grado de exposición de sus estructuras arqueológicas, así como por la presencia de elementos en pie en proceso de deterioro (Coahila, 2016). (Ver Tabla 3)

Respecto a este punto es importante aclarar que se han desarrollado trabajos de ampliación de sistemas de drenaje pluvial (canaletas, montantes) y a nivel de altura (ampliación de coberturas, drenaje suspendido, reforzamiento de estructuras), así como de habilitación de canales para evacuación de aguas subterráneas, y de protección de coberturas existentes. Estas actividades se han desarrollado en la etapa final de los trabajos de prevención.

\section{De la instalación de coberturas}

Como se menciona en el título de este acápite, la instalación de cubiertas permitió obtener muchas conclusiones sobre las áreas consideradas dentro de las acciones de prevención. Ello gracias al gran número de coberturas utilizadas, que permitió analizar su comportamiento sobre las estructuras que cobijaban y frente a diversos agentes climáticos que incidieron en ellas.

En la mayoría de las áreas de intervención se obtuvieron muy buenos resultados, especialmente en estructuras donde la interacción de factores de deterioro no fue muy variada, cumpliendo la finalidad a la que fue destinada. Así, también se pudo apreciar otro tipo de reacciones que llamaron bastante la atención, y que es importante destacar, debido al comportamiento que estas coberturas instaladas provocaron sobre los elementos que protegían.
Figura 5. Avanzado desgaste de las coberturas instaladas, colapsadas por falta mantenimiento y desgaste. Estos materiales deleznables fueron reemplazados por planchas de fibra vegetal.

Fuente: Archivo fotográfico personal Alberto Coahila, 2014. 
devenir Vol. 3, N55, ENERO - JUNIO 2016, PP. 90-112 - ESTUDIOS I ISSN 2312-7570

UNIVERSIDAD NACIONAL DE INGENIERÍ, LIMA

Tabla 3. Clasificación de las áreas de intervención de la segunda etapa

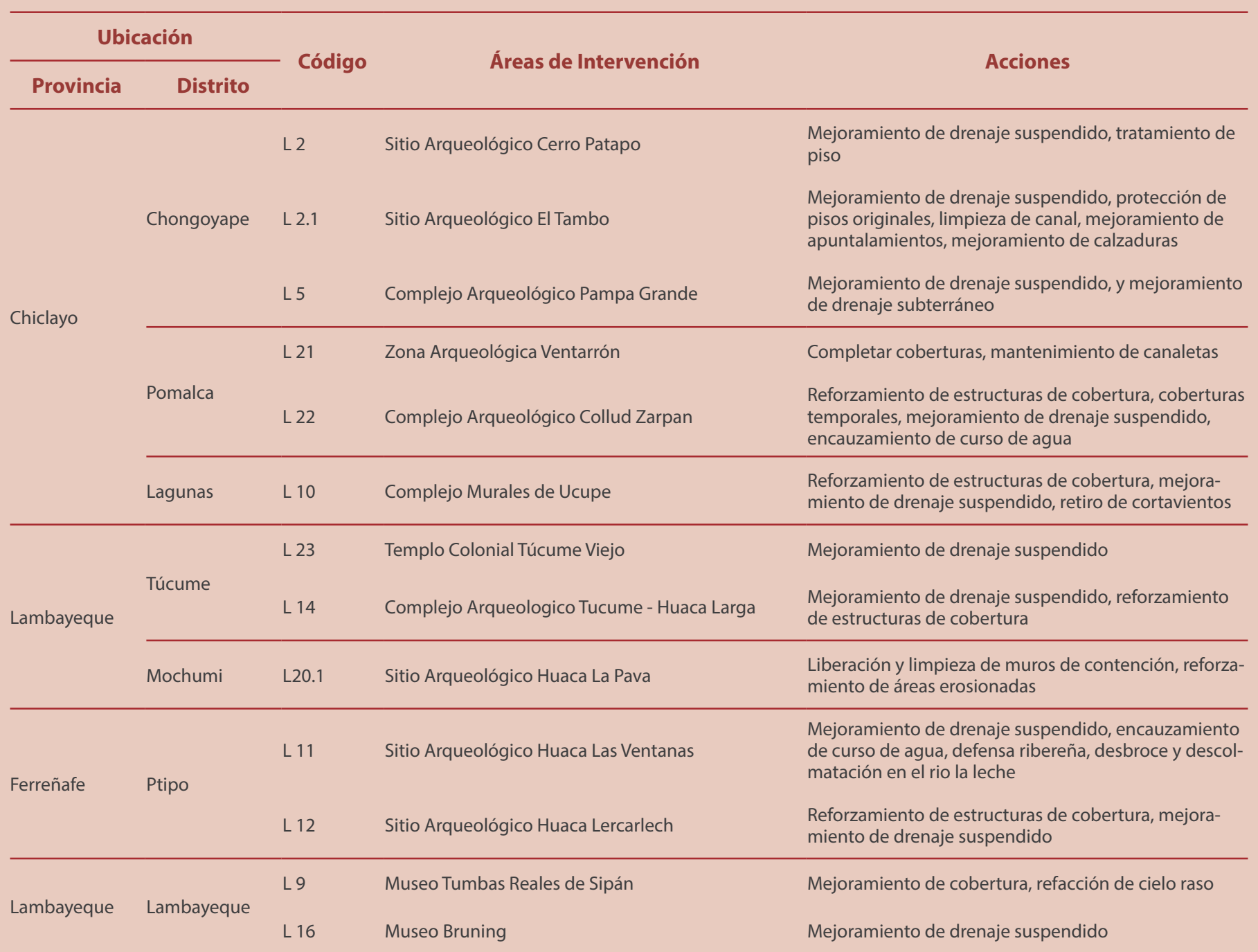

Fuente: Elaboración Alberto Coahila, 2014.

Este análisis corresponde a la cobertura instalada en la galería Este del claustro mayor del Convento San Agustín, ubicado en la localidad de Zaña. Los elementos a proteger fueron las bases de los pilares que aún quedan en pie, y que alcanzan una altura máxima de 1.2 metros, aproximadamente. Estos pilares se encuentran en avanzado proceso de deterioro debido a la humedad existente en el subsuelo, la cual, a través de la capilaridad, ha afectado las condiciones físicas de los materiales que las conforman. Ha provocado, así, la activación de las sales y ha propiciado un avanzado proceso erosivo de carácter físico en los materiales. Respecto a esto, es importante resaltar que los elementos a proteger no presentaban humedad antes de la instalación de la cobertura, pero sí mostraban un avanzado proceso de deterioro (ver Figuras 6 y 7 ).

Identificado este primer factor de deterioro, se procedió a determinar la fuente que originaba la fuerte humedad en este sector de la edificación, que había deteriorado 


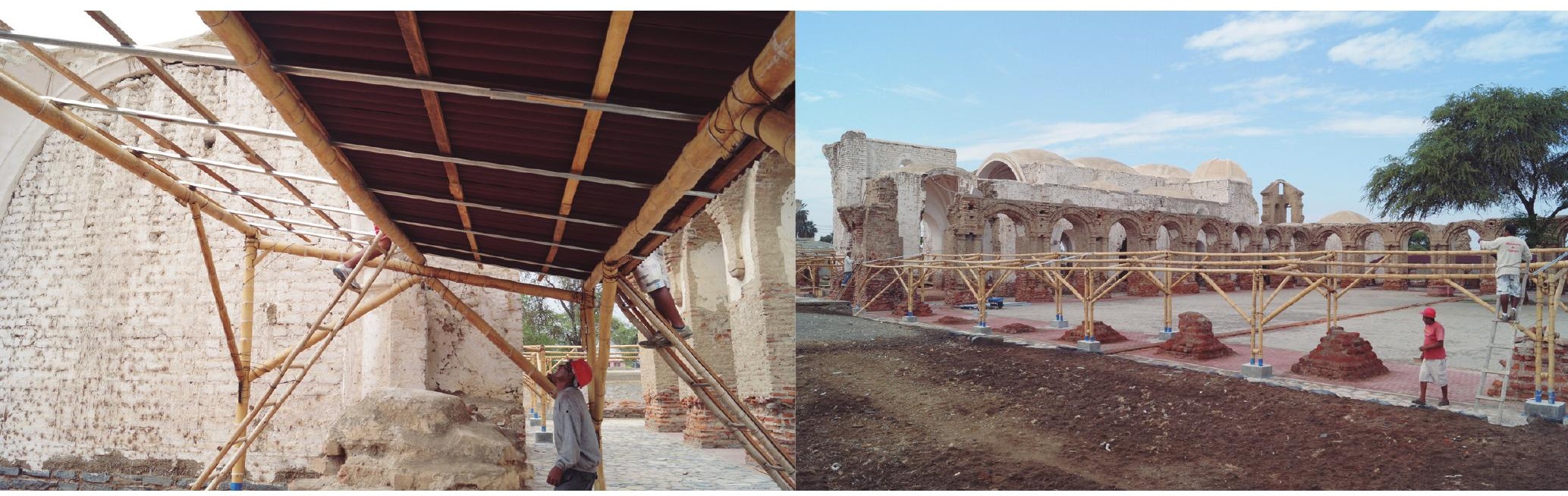

muy severamente las estructuras. Se llegó a corroborar lo elevado de la capa freática en este sector, la cual aumenta y disminuye su nivel según a los periodos de lluvias estacionarias presentes en la zona (ver Figura 8).

De acuerdo a la programación en la ejecución de los trabajos de prevención, se procedió, como primer paso, al montaje de la cobertura en este sector, donde se instaló una cubierta con una altura aproximada de 2.50 metros sobre los pilares. Concluidos los trabajos de prevención y pasado un corto tiempo, se iniciaron las acciones de monitoreo de los trabajos realizados, en las que se observó algo peculiar en el comportamiento de las estructuras protegidas con la cobertura colocada; de los siete pilares a cubrir, solo quedó expuesto uno, a la intemperie, ya que la falta de materiales no permitió completar el tramo faltante. Este imprevisto permitió dilucidar hipótesis, lo que abrió un abanico de posibilidades para un estudio posterior más minucioso (ver Figura 9).

Lo peculiar en este caso fue la reacción que tuvieron los pilares cubiertos en comparación al último pilar, que quedó sin ningún tipo de cobertura: los que contaban con protección mostraban un evidente aumento de áreas húmedas, en comparación al pilar que se encontraba a la intemperie, que no mostraba indicios de humedad en toda su extensión. Lo que se dedujo a simple vista fue que las áreas cubiertas no permitín eliminar de manera natural la humedad contenida en su interior. Este factor aceleró su proceso de deterioro, lo que no sucedía en el área expuesta, donde la radiación solar permitía eliminar por completo la presencia de humedad. Así, se llegó a deducir que un objeto, al estar en condiciones estables a las que ya se adaptó, se ve alterado de un momento a otro por la inserción de elementos ajenos, lo que provocó un cambio brusco en su ciclo normal y ocasiona reacciones adversas, que propician un daño en las cualidades internas de los materiales hasta llevarlos a un total e irreversible deterioro.

Este proceso permitió comprender que, para realizar estos trabajos, es muy importante tomar en cuenta consideraciones previas a la instalación de coberturas. En ese sentido, se debe

...realizar un análisis minucioso de las áreas a cubrir, conocer que factores inciden en el deterioro, evaluar la necesidad de su instalación y otros mecanismos de protección, monitoreando la reacción sobre las estructuras a proteger y entender el comportamiento frente a cambios tan bruscos ya que al pasar de un ambiente adaptado y en condiciones estables se ven alteradas de un momento a otro provocando este tipo de reacciones. (World Monuments Fund, Fundación Pedro Elena Hernándes A.C., Fomento Cultural Banamex, 2011) (ver Figura 10)
Figura 6. Convento de San Agustín de Zaña: instalación de la plancha de fibra vegetal como material de cubierta en el sector Oeste del claustro principal

Fuente: Archivo fotográfico Unidad Ejecutora 005 Naylamp Lambayeque, 2014

Figura 7. Convento de San Agustín de Zaña: instalación de coberturas sobre los pilares de la galería Este, los cuales tuvieron un comportamiento distinto a otras áreas por la presencia de humedad en el subsuelo

Fuente: Archivo fotográfico Unidad Ejecutora 005 Naylamp Lambayeque, 2014. 


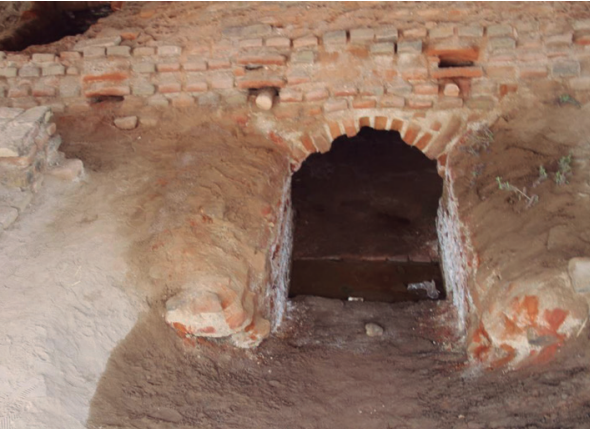

Figura 8. Sectores contiguos a la galería Este. Existió un aumento de agua que llegaba hasta una altura de $20 \mathrm{~cm}$, generando la presencia de humedad en los pilares cubiertos

Fuente: Archivo fotográfico personal Alberto Coahila, 2014.

Figura 9. Vista del pilar sin cobertura, donde se observó un comportamiento distinto al sus pares con cobertura instalada

Fuente: Archivo fotográfico Unidad Ejecutora 005 Naylamp Lambayeque, 2014.

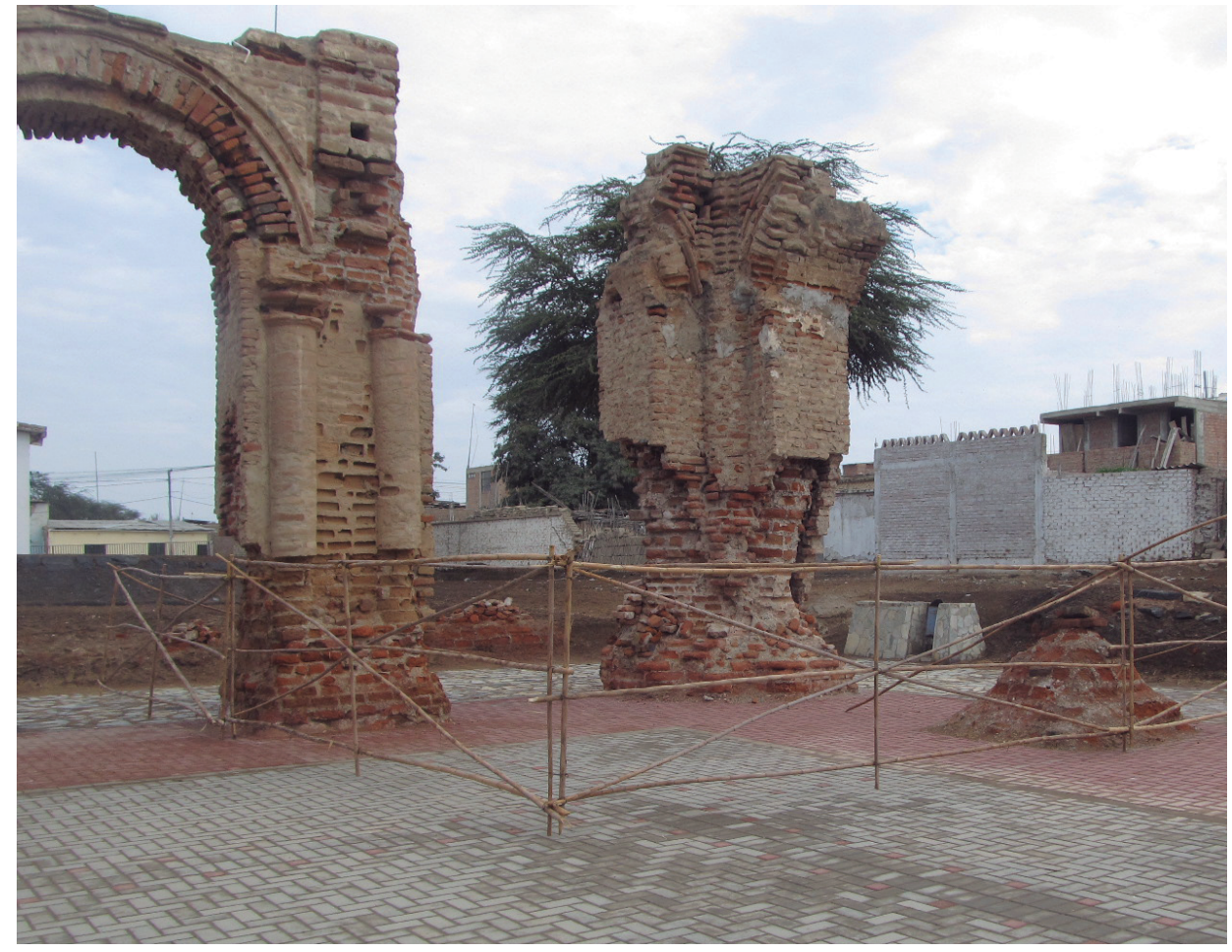

Por lo tanto, estos trabajos de prevención dejaron muchas lecciones a tomar en cuenta, especialmente en la instalación de coberturas, donde las acciones de corrección de errores a corto plazo permitieron obtener resultados adecuados en la mayor parte. En ese sentido, la protección del patrimonio arqueológico, como cualquier otra acción, requiere de un trabajo planificado, que permita programar acciones de manera sistemática con el fin de lograr mejores resultados. Así, es importante proyectar acciones de protección de manera planificada para atender a otros sectores con alto riesgo de sufrir daños, con la finalidad de enfrentar de mejor manera los cambios climáticos que pueden manifestarse en determinadas épocas del año.

En función a lo mencionado, la Unidad Ejecutora 005 Naylamp - Lambayeque, encargada de la protección, investigación, conservación y puesta en valor del patrimonio arqueológico, paralelamente viene desarrollando actividades para poder obtener información sobre el estado actual de los sitios arqueológicos y plantear estrategias de actuación en toda la región de Lambayeque. Esto a través de la elaboración de un diagnostico físico espacial, como un primer componente dentro de la elaboración de un Plan Maestro, en la actualidad en proceso de realización, para todos los sitios arqueológicos con los que cuenta la región. Este diagnóstico ha permitido determinar de manera integral los factores que inciden en el estado actual de conservación del patrimonio cultural prehispánico.

Asimismo, este diagnóstico tuvo la finalidad de reconocer el entorno donde se ubican los distintos sitios arqueológicos, mediante una metodología de concepto territorial y de paisaje. El mismo ha proporcionado las líneas base sobre las diversas problemáticas y el grado de vulnerabilidad frente a diversos factores que pudieran afectar al patrimonio cultural arqueológico. Para esto, uno de los criterios a tomar en cuenta fue la configuración geomorfológica de la región, que sirvió para conformar unidades de paisaje a fin de obtener un mejor conocimiento sobre la problemática y manejo de las áreas donde se ubican (ver Figura 11). 


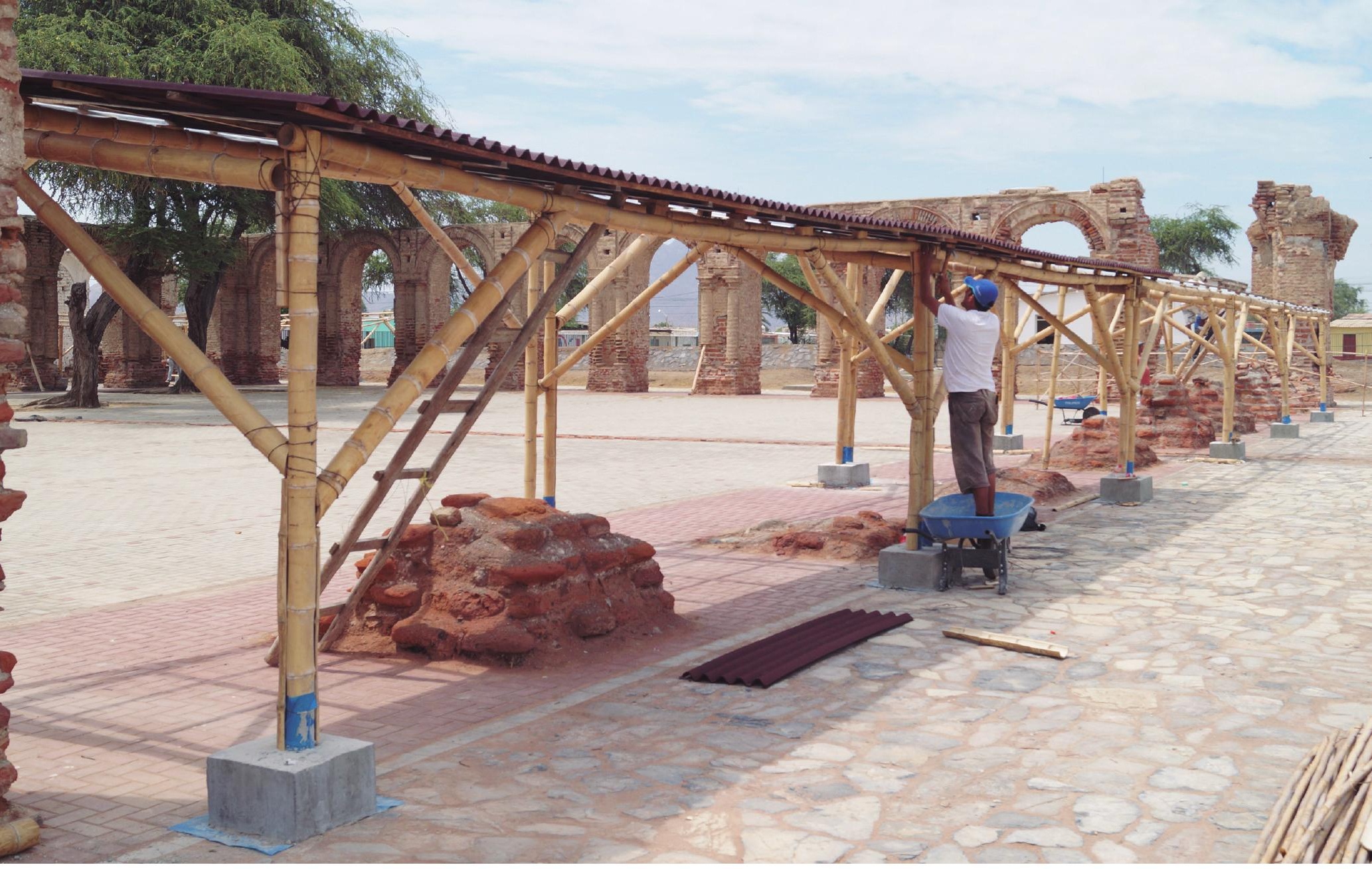

Esta rica historia de ocupación y transformación del territorio se evidencia en las abundantes evidencias arqueológicas del período prehispánico. La cifra del registro correspondiente al número total de sitios arqueológicos de los que tiene constancia actualmente la UE 005 es de 736 sitios arqueológicos; una segunda cifra refiere a una muestra de 108 sitios arqueológicos en los que se ha evaluado la problemática que los afecta. (Martínez, 2015). Las afectaciones por causas naturales son una dinámica de los procesos ambientales propios de la región de la costa norte del Perú (ver Figura 12).

\section{Resultados de la evaluación}

Los resultados de la evaluación realizada durante los trabajos de campo permitieron conocer la configuración físico espacial de la región Lambayeque, con la finalidad de tener un mejor manejo y monitoreo de las áreas identificadas con diferente nivel de riesgo frente a diversos factores que pudieran afectar a los sitios arqueológicos en estas áreas.

Como se sabe, las afectaciones más generalizadas han sido provocadas por causas naturales, consecuencia del proceso climático propio de la costa norte del Perú.

La amplia llanura costera, con su secuencia de valles fértiles y extensos desiertos con elevadas dunas, y las afectaciones periódicas del Fenómeno de El Niño (ENSO), que desencadena lluvias torrenciales, que a su vez activan quebradas y provocan deslizamientos e inundaciones, están en la base de las dinámicas naturales de afectación de los sitios arqueológicos. Así lo señala también Martínez (2015):

...los efectos de estas condiciones naturales se pueden intensificar en situación de cambio climático, pues la costa norte será uno de los espacios que sufrirá mayores afectaciones con una intensificación y acortamiento de los ENSO, que obligaran a tomar acciones preventivas más permanentes y menos excepcionales. (p. 12) (Ver Tabla 4)
Figura 10. Notoria presencia de humedad en pilares cubiertos. Se pudo entender que la cobertura instalada alteró el ciclo de secado de estos elementos, en comparación al que quedó descubierto, donde la incidencia de los rayos solares eliminaba todo rastro de humedad y desaceleraban el proceso de deterioro

Fuente: Archivo fotográfico Unidad Ejecutora 005 Naylamp Lambayeque, 2014. 


\section{Peligros a los que está expuesto el patrimonio arqueológico}

Como ya se señaló, una de las características más importantes de la región Lambayeque, en cuanto a peligros de causa natural, es la alta actividad geodinámica, que se evidencia principalmente durante el Fenómeno de El Niño:

Los documentos históricos muestran también que la geodinámica interna ha afectado muchos centros poblados en siglos pasados. Los procesos de ocupación y la sobreexplotación de los recursos naturales, así como la construcción de infraestructuras, contribuyen a la desestabilización de muchos sistemas geomorfológicos, evidenciándose en la ocurrencia de movimientos en masa, la mayor intensidad de los procesos erosivos y la ocurrencia de inundaciones en zonas donde de manera natural son poco frecuentes. Los peligros considerados con potencial afectación al patrimonio arqueológico son los peligros geomorfológicos (peligros hidromorfológicos y movimientos en masa. (Martínez, 2015, p. 48)

Estos peligros están vinculados principalmente con las anomalías climáticas. Los peligros considerados en esta categoría son las inundaciones y flujos torrenciales (flujos de lodo y huaycos); son fenómenos de rápido desarrollo que han causado gran afectación en el pasado. En la región Lambayeque los fenómenos hidromorfológicos más evidentes son las inundaciones, esto principalmente porque han afectado áreas agrícolas, centros poblados e infraestructura. La activación de las cuencas con cauces torrenciales y el aumento del caudal de los ríos. (Martínez, 2015, p. 49)

A continuación se muestra la relación de sitios inventariados de manera general dentro de la elaboración del diagnóstico territorial del Plan Maestro ubicados en las cuencas hidrográficas de la región (Ver Tabla 5 y Figura 13).

Actualmente, las dinámicas territoriales son condicionadas por la consolidación y el crecimiento poco planificado, así como expansivo, de los espacios urbanos, y el avance de la frontera agrícola, especialmente hacia las tierras eriazas y piedemonte, lo cual se suma a la lista de factores que inciden en el deterioro del patrimonio en la región. También son un factor las actividades extractivas:

Las actividades extractivas (canteras de material pétreo) tanto formales como informales generan grandes afectaciones al paisaje por ende alteran los contextos tan frágiles donde se emplazan las estructuras arqueológicas, la contaminación ambiental con desechos en suspensión trasladados por las corrientes de vientos y la contaminación producida por la quema de vegetación viene provocado daños reversibles. (Martínez, 2015, p. 8)

Las actividades extractivas, básicamente canteras de piedra se han incrementado con el auge de la construcción, muchas de ellas son informales, como otras que cuentan con permisos para desarrollar sus actividades de manera formal, inclusive en áreas con abundantes sitios arqueológicos registrados. Así mismo las municipalidades también recurren a la extracción de materiales de los cerros durante los periodos de lluvias, especialmente en eventos lluviosos con la finalidad de poder realizar trabajos de reforzamiento ejecutando obras de protección ante el traslado de flujos de materiales como avalanchas e inundaciones. Es por ello que muchos sitios arqueológicos se encuentran afectados. (Martínez, 2015, p. 9)

Otras de las principales afectaciones, como es de amplio conocimiento, se produce debido a las acciones de huaqueo, seguido por el arrojo de basura y desmonte, y por el desarrollo de la pequeña y mediana actividad agrícola. La expansión vial, la expansión de los asentamientos de población en ámbitos no urbanos, así como la infraestructura energética y de riego, también constituyen afectaciones que se observan en más del $30 \%$ de los sitios arqueológicos. Sobre estos factores de afectación, Martínez señala lo siguiente:

La utilización del patrimonio como vertedero de basura y desmonte afecta en buena parte de los sitios arqueológicos, especialmente los que se encuentran en ámbitos urbanos y cerca a centros poblados, esto se debe a la ausencia de políticas de carácter ambiental de 
ámbito regional y municipal que brinde las directrices sobre la disposición de los residuos urbanos y el desmonte. $(2015$, p. 8)

El boom inmobiliario ha traído efectos perniciosos en el destino de los desmontes generados por esta actividad, dejados impunemente en los bordes de los caminos y también en sitios arqueológicos, generando contaminación sobre las estructuras arqueológicas y también al medio ambiente. La agricultura es la tercera causa de afectación al patrimonio arqueológico. El tipo de cultivos que afecta de manera diferencial al patrimonio, así el cultivo del arroz propio de los pequeños y medianos agricultores, por el encharcamiento de las tierras es lo que trae más daños a los monumentos provocando áreas de intensa humedad lo que conlleva a un debilitamiento de las bases y toda estructura donde llega provocando proceso erosivos severos a consecuencias de los proceso de salinización que se inician una vez que entren en contacto con el agua. $(2015$, p. 8$)$

Por lo tanto, se puede identificar situaciones comunes, como el uso agrícola al borde mismo del monumento, que por su proximidad a este incrementa el riesgo de afectaciones derivadas del riego o de operaciones agrícolas con maquinaria. El único mecanismo de defensa frente a esto es la delimitación arqueológica, tema en el que aún queda mucho por realizar, que sin embargo, de alguna manera ha ayudado a revertir esta situación, cuyo impacto causaría la pérdida de la memoria histórica de manera irreparable.

La infraestructura vial es otro factor muy importante de daño. El criterio con el que se han trazado las vías ha sido las necesidades de conectividad de asentamientos o de espacios de producción, por lo que en la mayoría de casos no se ha tomado en cuenta la ubicación de los sitios arqueológicos.

La falta de integración y la ausencia de un trabajo que tome en cuenta todos los actores involucrados dentro de un contexto tan vulnerable, y la carencia de una priorización de las necesidades, se tornan en la base de la destrucción histórica en esta región.

Como se menciona en los párrafos anteriores, los daños al patrimonio en la región Lambayeque obedecen a varios factores, los cuales actúan como elementos catalizadores al interactuar entre ellos, que acrecientan y aceleran la pérdida de los numerosos sitios con un importante valor cultural. Se observa cómo los fenómenos climáticos recurrentes en ciertas épocas de año provocan otras reacciones, como la activación de cuencas, que modifica la configuración morfológica del territorio. Esto se traduce en la modificación de las formas de ocupación poblacional en muchos sectores, con acciones no planificadas que generan nuevos asentamientos en áreas más elevadas, las cuales, coincidentemente, corresponden a los sitios arqueológicos. Otro de los daños generados por los fenómenos naturales son los desbordes de canales de riego y ríos, lo que provoca inundaciones. Respecto a esto, la población afectada, para salvaguardar su integridad y la de los poblados, generan nuevos cauces con la finalidad de mitigar este daño, sin tomar en cuenta las consecuencias que pueden causar estos nuevos cursos de agua. Muchos de ellos pasan por áreas arqueológicas, arrasando todo a su paso, por lo que destruyen estas áreas y las afectan irreversiblemente.

Es evidente que, si bien se pueden mitigar los daños producidos por los efectos de la naturaleza, la tarea se torna más complicada aun cuando se trata de factores antrópicos. Los procesos de ocupación urbana, la expansión de la frontera agrícola, las acciones de huaqueo que ocurren de manera permanente, la acumulación de basura y desmonte, así como la quema de cultivos, se han convertido en un problema incontrolable. Por ello es importante trabajar de manera conjunta con todos los actores y así poder tomar medidas de manera integral, que busquen satisfacer intereses primordiales, como son fortalecer la cultura a través de la territorialidad y el sentido de pertenencia en la población, a fin conseguir una mejor calidad de vida y la perdurabilidad del legado histórico edificado. 
devenir Vol.3, N5, ENERO- JUNIO 2016, PP. 90-112 - ESTUDIOS I ISSN 2312-7570

UnIVERSIDAD NACIONAL DE INGENIERÍA, LIMA

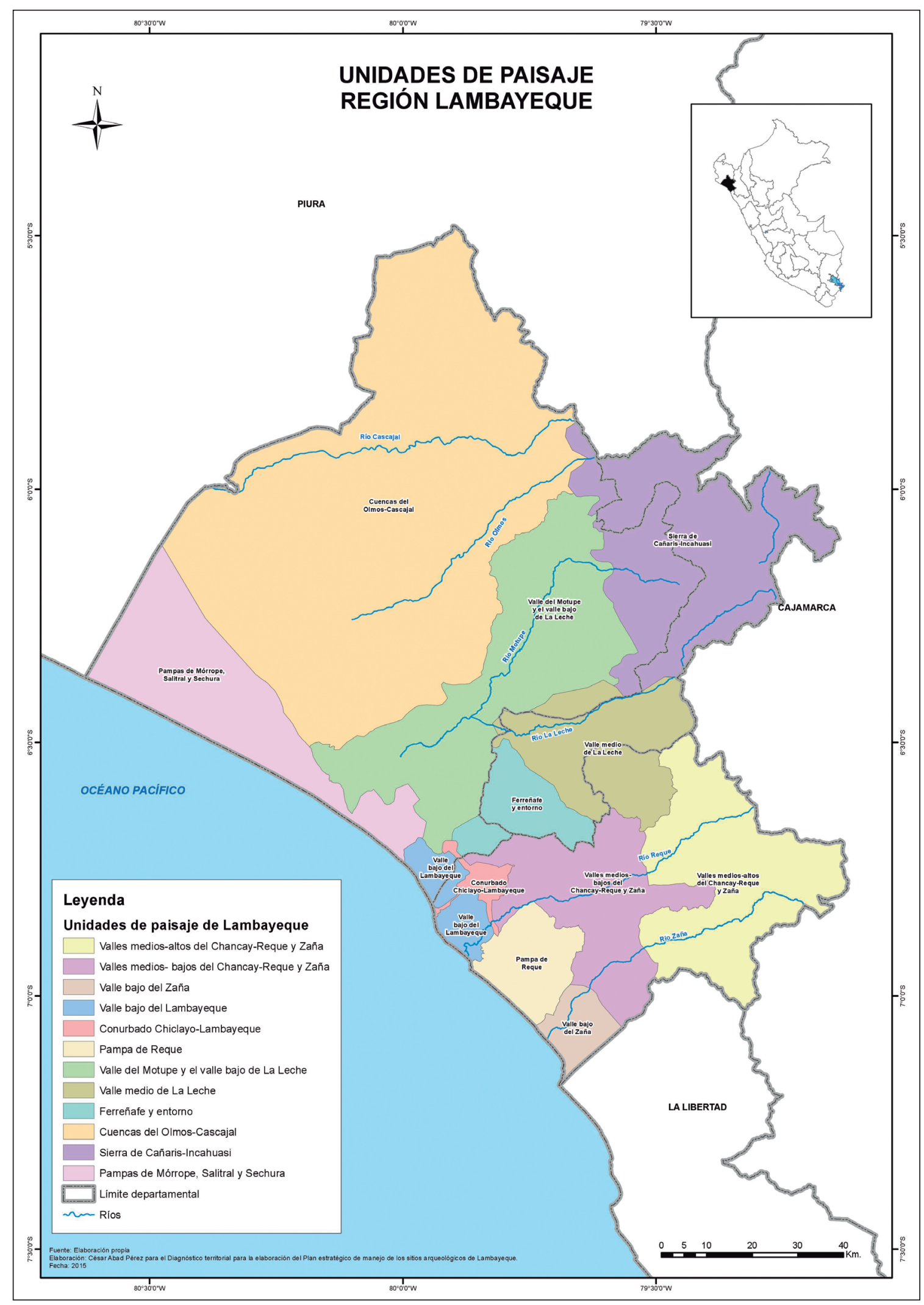

Figura 11. Unidades de paisaje de la región Lambayeque, como resultado del diagnóstico físico espacial Fuente: Martínez, 2015. 


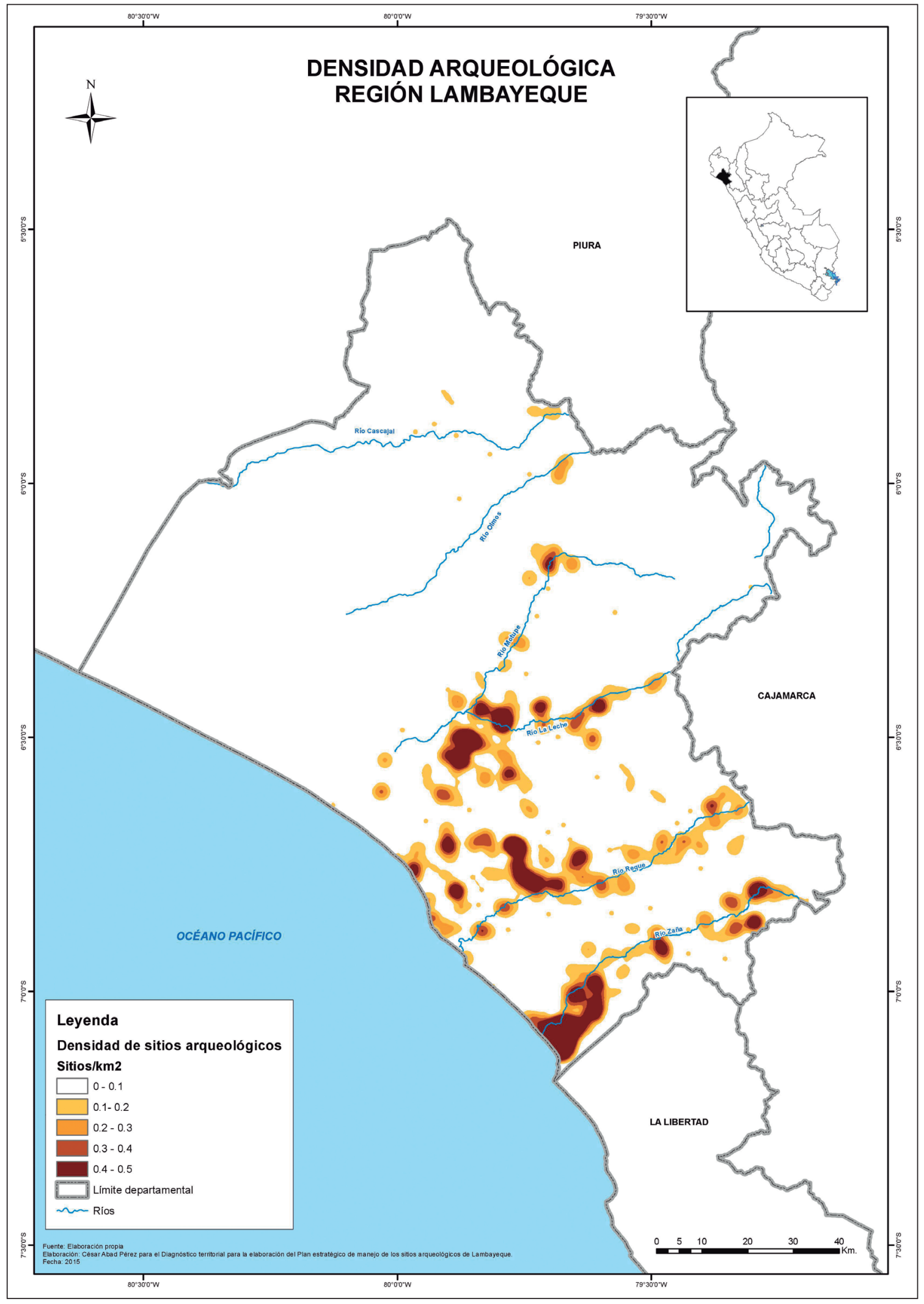

Figura 12. Densidad arqueológica de la Región Lambayeque Fuente: Martínez, 2015. 
devenir Vol. 3, N5, ENERO - JUNIO 2016, PP. 90-112 - EstUdIOS I ISSN 2312-7570

UNIVERSIDAD NACIONAL DE INGENIERÍ, LIMA

Tabla 5. Cuadro de factores y porcentaje de afectaciones. Mayor incidencia de las Iluvias, las cuales han provocado daño en las zonas arqueológicas de la región Lambayeque Fuente: Martínez, 2015.

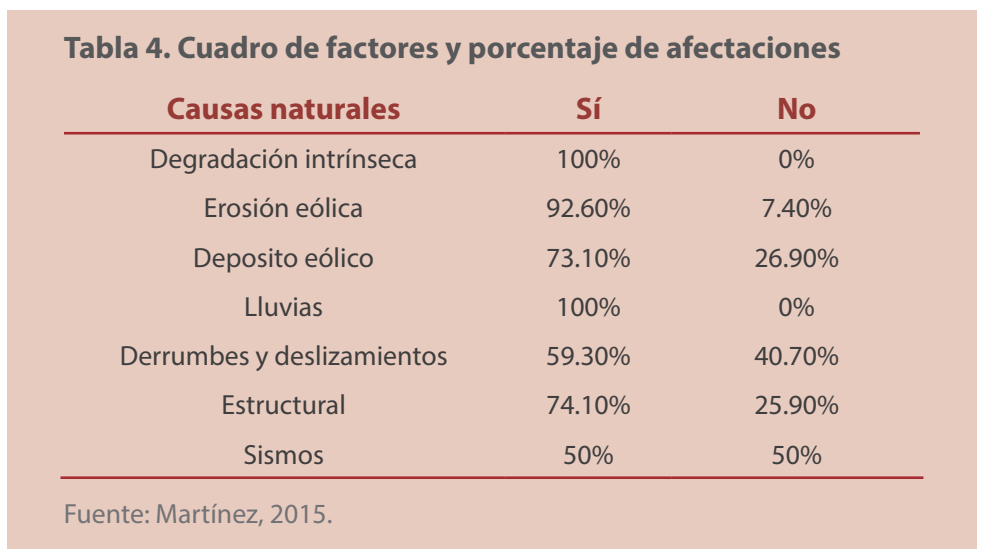

\section{Medidas implementadas}

El diagnóstico físico-espacial, desarrollado como un primer componente para la elaboración del Plan Estratégico de Manejo de los Sitios Arqueológicos de Lambayeque que la UE005 viene desarrollando, permite programar acciones de prevención, cuya finalidad es garantizar la protección de la mayoría de los sitios arqueológicos inventariados en la región Lambayeque.

Por ello es necesario priorizar las intervenciones de acuerdo a criterios definidos, que se detallan a continuación:

(A) Debido al gran número de patrimonio arqueológico con que cuenta la región, las actividades a programar se deben orientar, en primer lugar, a los sitios arqueológicos en proceso de investigación y que se encuentren vulnerables a la intemperie durante todo el año. Por lo tanto, la instalación de cubiertas provisionales para proteger las estructuras y superficies policromadas es una medida de protección importante en estos casos.

(B) Sin embargo, la mayoría de sitios arqueológicos de esta región aún no han sido intervenidos y deben ser protegidos de manera indirecta a fin de prevenir desastres futuros. Las medidas de intervención estarán dirigidas, básicamente, a realizar acciones de limpieza y descolmatación de cauces de río que pasen cerca de las estructuras arqueológicas, las que se encuentran distribuidas en toda la margen de los ríos que conforman las cuencas hidrográficas de la región, así como otras actividades de limpieza de quebradas, instalación de muros de contención y de defensas ribereñas en sectores que sea necesario intervenir.

En relación a la presencia del fenómeno de El Niño, sea cual sea su magnitud, se deberán programar actividades con la finalidad de proteger los sitios arqueológicos, mediante el desarrollo de acciones con anticipación, y que tengan como referencia la ocurrencia de este fenómeno en 1982-83 y 1997-98. Estos eventos han permitido desarrollar un programa de prevención: el Marco de Desarrollo de las Actividades.

Frente a este panorama que se viene presentando, junto a la información del diagnóstico físico espacial, y con el conocimiento más minucioso de la incidencia de los factores que afectan en el estado de conservación de los sitios arqueológicos, se plantea un "Plan de Acción Inmediata ante la probable ocurrencia del Fenómeno El Niño 2015- 2016". En este se puede identificar que el mayor daño puede ser producido por los flujos o corrientes de agua que discurrirán a lo largo de todo el recorrido de las cuencas, que provienen de las cabeceras de la cadena montañosa (quebradas y cauces secos), lo cual producirá inundaciones y desbordes, principales factores de deterioro de los sitios arqueológicos. 


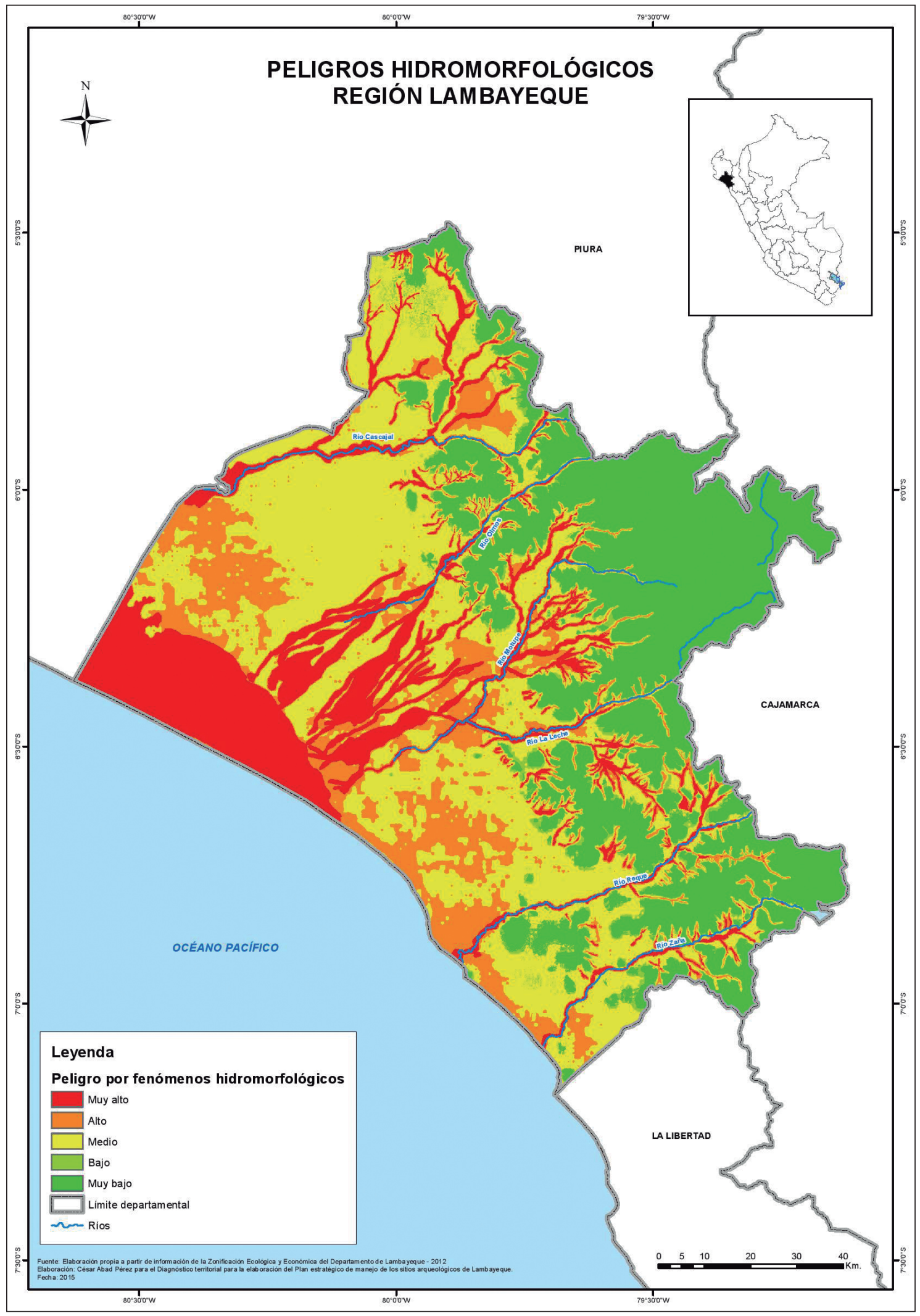

Figura 13. Peligros hidromorfológicos en la región Lambayeque Fuente: Martínez, 2015. 
devenir Vol. 3, N5, ENERO - JUNIO 2016, PP. 90-112 - ESTUDIOS I ISSN 2312-7570

UNIVERSIDAD NACIONAL DE INGENIERÍA, LIMA

Tabla 5. Identificación de los sitios arqueológicos en las cuencas hidrográficas de la región Lambayeque Sector cuenca hidrográfica Olmos

\begin{tabular}{ccccccc} 
& Sitio Arqueológico & Lluvias & Derrumbes $\mathbf{y}$ & & \multicolumn{2}{c}{ Grado de afectación } \\
\cline { 5 - 7 } & deslizamientos & Alta & Media & Baja \\
\hline 1 & La Huaca 1 & $\mathrm{Si}$ & $\mathrm{Si}$ & $\mathrm{X}$ & \\
2 & Tres Batanes & $\mathrm{Si}$ & $\mathrm{No}$ & $\mathrm{X}$ & \\
3 & San Pedro de Racalín & $\mathrm{Si}$ & $\mathrm{Si}$ & $\mathrm{X}$ & & \\
4 & Cerro Mano de León & $\mathrm{Si}$ & $\mathrm{No}$ & & $\mathrm{X}$ & $\mathrm{X}$ \\
5 & Quebrada Los Boliches & $\mathrm{Si}$ & $\mathrm{No}$ & & &
\end{tabular}

Fuente: Martínez, 2015.

Sector cuenca hidrográfica Lambayeque

\begin{tabular}{|c|c|c|c|c|c|c|}
\hline \multirow{2}{*}{$\mathbf{N}^{\circ}$} & \multirow{2}{*}{ Sitio Arqueológico } & \multirow{2}{*}{ Lluvias } & \multirow{2}{*}{$\begin{array}{l}\text { Estructural (fractu- } \\
\text { ra, falla) }\end{array}$} & \multicolumn{3}{|c|}{ Grado de afectación } \\
\hline & & & & Alta & Media & Baja \\
\hline 1 & San José 1 & $\mathrm{Si}$ & $\mathrm{Si}$ & $x$ & & \\
\hline 2 & San José 2 & $\mathrm{Si}$ & $\mathrm{Si}$ & $x$ & & \\
\hline 3 & San José 3 & $\mathrm{Si}$ & Si & $x$ & & \\
\hline 4 & San José 4 & $\mathrm{Si}$ & $\mathrm{Si}$ & $\mathrm{x}$ & & \\
\hline 5 & Huaca Blanca Norte & $\mathrm{Si}$ & $\mathrm{Si}$ & & $x$ & \\
\hline 6 & Huaca Mirador Bodegones & $\mathrm{Si}$ & $\mathrm{Si}$ & $x$ & & \\
\hline 7 & Complejo Arqueológico Mocce & $\mathrm{Si}$ & Si & $x$ & & \\
\hline 8 & Huaca Santa Rosa 01 & $\mathrm{Si}$ & $\mathrm{Si}$ & $x$ & & \\
\hline 9 & Huaca Santa Rosa 02 & $\mathrm{Si}$ & $\mathrm{Si}$ & $x$ & & \\
\hline 10 & Huaca Santa Rosa 03 & $\mathrm{Si}$ & Si & $x$ & & \\
\hline 11 & Huaca La Tina & Si & Si & & $x$ & \\
\hline 12 & Huaca Cercado Lambayeque & Si & Si & $x$ & & \\
\hline 13 & Huaca Los Perros & $\mathrm{Si}$ & $\mathrm{Si}$ & & $x$ & \\
\hline 14 & Huaca Sialupe & Si & Si & $x$ & & \\
\hline 15 & Complejo Arqueológico Chotuna - Chornancap & $\mathrm{Si}$ & $\mathrm{Si}$ & & & $x$ \\
\hline
\end{tabular}

Fuente: Martínez, 2015.

Sector cuenca hidrográfica Zaña

\begin{tabular}{|c|c|c|c|c|c|c|}
\hline \multirow{2}{*}{$\mathbf{N}^{\circ}$} & \multirow{2}{*}{ Sitio Arqueológico } & \multirow{2}{*}{ Lluvias } & \multirow{2}{*}{$\begin{array}{l}\text { Derrumbes y desli- } \\
\text { zamientos }\end{array}$} & \multicolumn{3}{|c|}{ Grado de afectación } \\
\hline & & & & Alta & Media & Baja \\
\hline 1 & Complejo Úcupe Pueblo & $\mathrm{Si}$ & No & & $x$ & \\
\hline 4 & Pampas de Mata Indio & $\mathrm{Si}$ & No & $x$ & & \\
\hline 7 & Huaca Murales de Úcupe & $\mathrm{Si}$ & No & & $x$ & \\
\hline 8 & Paitora & No & No & & & \\
\hline 9 & Yanya & No & No & & $x$ & \\
\hline 17 & Huaca Rajada Sipán & $\mathrm{Si}$ & $\mathrm{Si}$ & & $x$ & \\
\hline 18 & Cementerio Sipán & $\mathrm{Si}$ & No & & $x$ & \\
\hline
\end{tabular}

Fuente: Martínez, 2015. 
Por lo tanto, es necesario contar con mayor presupuesto para realizar estas actividades de prevención, cuya programación fue resultado de las evaluaciones realizadas con la finalidad de cubrir la emergencia en los sitios designados con mayor prioridad, debido a la fuerte amenaza ambiental que este evento climático representa para estos.

Durante la presencia de este fenómeno climático, una asignación presupuestal considerable servirá para programar la atención de los sitios arqueológicos según las premisas detalladas en los párrafos (A) y (B), con la finalidad de enfrentar de mejor manera los daños que provocará este evento climático. Para este fin, se deben programar de manera anticipada las acciones a tomar, considerando los sectores de intervención a través de un mapeado general, en el que se identifiquen sectores de directa incidencia que tengan en cuenta que este fenómeno se presenta de manera cíclica y con mayor impacto en la región Lambayeque.

\section{Lecciones aprendidas}

Dentro de las acciones de prevención por el fenómeno de El Niño, ejecutadas sobre el patrimonio arqueológico en la región Lambayeque, no se realizó una correcta evaluación de riesgos de los monumentos expuestos al constante deterioro por eventos naturales. Estos eventos han ocasionado el debilitamiento de las estructuras arquitectónicas y producido, en algunos casos, el desplome de la arquitectura visible y, en la mayoría de los casos, la erosión de los montículos arqueológicos. Por ello, es importante hacer un análisis desde la temporalidad de las estructuras arqueológicas, que por su antigüedad de más de 3000 años, muestran en la actualidad la fragilidad de sus estructuras, por lo que son necesarias e indispensables las acciones de prevención integrales, planteadas dentro de las actividades de conservación y restauración. Así, se debe

... considerar la gran relevancia de los diferentes sitios arqueológicos puesto que representa la historia cultural de la zona norte del país deben de ser protegidos considerando el grado de avance referido a su progresivo deterioro por la presencia de lluvias, por lo tanto es importante realizar un análisis de vulnerabilidad que permita entender aspectos como el grado de exposición. (Laínez, 2015, p. 6)

Es importante tener en cuenta lo delicado que resulta realizar este tipo de trabajos en contextos tan frágiles, ya sea por el estado delicado de conservación de sus estructuras o por la información que guardan sus elementos. Esta se podría perder si no se manejan de manera adecuada los trabajos de prevención; por lo tanto, es importante considerar un monitoreo arqueológico continuo durante el desarrollo de estos trabajos, a fin de salvaguardar la integridad de las estructuras. "Asimismo, se debe tener un conocimiento del contexto y su comportamiento frente a estos eventos climáticos, a través de un análisis morfológico del área donde se emplazan las estructuras a proteger. Esto con la finalidad de que las soluciones planteadas funcionen de manera óptima, de acuerdo a lo planificado, y no se conviertan en factores de deterioro que aceleren la perdida de este legado..." (Coahila, Plan de acción inmediata ante la probable ocurrencia del fenómeno El Niño 2015- 2016, 2015).

Respecto a esto último, fue importante conocer el historial de eventos anteriores sobre el fenómeno de El Niño, pues permitió identificar el grado de vulnerabilidad de los sitios arqueológicos, al reconocer las áreas inundables, el comportamiento de las quebradas, la crecida de cursos de agua y canales, entre otros aspectos.

La instalación de coberturas de protección se implementó con la intención de preservar dichas estructuras arqueológicas. Sin embargo, por la premura de su instalación y la cercanía del periodo lluvioso, no se elaboraron estudios pormenorizados e integrales sobre el impacto que estas pueden ocasionar al ser instaladas sobre las estructuras arqueológicas. En primer lugar, es importante reconocer el proceso de degradación que sufren los materiales que conforman algunas cubiertas, las cuales propician por sí mismas proce- 
devenir Vol. 3, Nº5, ENERO - JUNIO 2016, PP. 90-112 - ESTUDIOS I ISSN 2312-7570

UnIVERSIDAD NACIONAL dE INGENIERÍA, LIMA

\begin{tabular}{|c|c|c|c|c|c|c|}
\hline \multirow{3}{*}{$\mathbf{N}^{\circ}$} & \multicolumn{6}{|c|}{ Sector cuenca hidrográfica Chancay - Reque } \\
\hline & \multirow{2}{*}{ Sitio Arqueológico } & \multirow{2}{*}{ Lluvias } & \multirow{2}{*}{$\begin{array}{l}\text { Derrumbes y desli- } \\
\text { zamientos }\end{array}$} & \multicolumn{3}{|c|}{ Grado de afectación } \\
\hline & & & & Alta & Media & Baja \\
\hline 1 & Pozo loco & $\mathrm{Si}$ & No & $\mathrm{x}$ & & \\
\hline 2 & Chacupe & $\mathrm{Si}$ & No & $x$ & & \\
\hline 3 & Los Huaquitos & $\mathrm{Si}$ & No & $\mathrm{x}$ & & \\
\hline 4 & Los Gentiles & $\mathrm{Si}$ & No & $x$ & & \\
\hline 5 & Montículo Las Rocas & $\mathrm{Si}$ & No & & $x$ & \\
\hline 6 & Morro Etén & $\mathrm{Si}$ & $\mathrm{Si}$ & & $x$ & \\
\hline 7 & Playa Los Lobos & $\mathrm{Si}$ & No & & $x$ & \\
\hline 8 & Huaca César Vallejo & $\mathrm{Si}$ & No & $x$ & & \\
\hline 9 & Huaca I.E. San José & $\mathrm{Si}$ & No & $x$ & & \\
\hline 10 & Cerro Cerrillos & $\mathrm{Si}$ & $\mathrm{Si}$ & $x$ & & \\
\hline 11 & Huaca Miraflores & $\mathrm{Si}$ & $\mathrm{Si}$ & $x$ & & \\
\hline 12 & Huaca El Taco & $\mathrm{Si}$ & $\mathrm{Si}$ & $x$ & & \\
\hline 14 & Huaca Epsel-Picsi (M4-Sector Norte) & $\mathrm{Si}$ & No & $x$ & & \\
\hline 15 & Huaca El Cementerio & $\mathrm{Si}$ & No & $x$ & & \\
\hline 16 & Huaca Oeste M-4 & $\mathrm{Si}$ & No & $x$ & & \\
\hline 17 & Huaca del Penal & $\mathrm{Si}$ & No & $x$ & & \\
\hline 18 & Huaca Picsi s/n & $\mathrm{Si}$ & No & $x$ & & \\
\hline 19 & Huaca San Juan 1 & $\mathrm{Si}$ & $\mathrm{Si}$ & & $x$ & \\
\hline 20 & Casa de Madera & $\mathrm{Si}$ & $\mathrm{Si}$ & & $x$ & \\
\hline 21 & Huaca Abejería & $\mathrm{Si}$ & $\mathrm{Si}$ & & $x$ & \\
\hline 22 & Cerro Combo & & & & & \\
\hline 23 & El Chorro & $\mathrm{Si}$ & $\mathrm{Si}$ & $x$ & & \\
\hline 24 & Complejo Collud-Zarpán & $\mathrm{Si}$ & $\mathrm{Si}$ & & $\mathrm{x}$ & \\
\hline 25 & Cerro Ventarron & $\mathrm{Si}$ & $\mathrm{Si}$ & & $x$ & \\
\hline 26 & Cementerio Pomalca & $\mathrm{Si}$ & $\mathrm{Si}$ & $x$ & & \\
\hline 27 & Luya & $\mathrm{Si}$ & $\mathrm{Si}$ & $x$ & & \\
\hline 33 & Santa Rosa & $\mathrm{Si}$ & No & & $x$ & \\
\hline 34 & Tucu Tucu & $\mathrm{Si}$ & No & $x$ & & \\
\hline 35 & La Inmaculada & $\mathrm{Si}$ & No & $x$ & & \\
\hline 36 & Algarrobal & $\mathrm{Si}$ & No & $x$ & & \\
\hline 37 & Huaca Vera & $\mathrm{Si}$ & No & $x$ & & \\
\hline 38 & Cerro Caballo Blanco & $\mathrm{Si}$ & $\mathrm{Si}$ & $x$ & & \\
\hline 39 & San Juan de la Punta & $\mathrm{Si}$ & No & & $x$ & \\
\hline 40 & Complejo Saltur & $\mathrm{Si}$ & No & & $x$ & \\
\hline 41 & Montículo Santa Rosa 1 & $\mathrm{Si}$ & No & & $x$ & \\
\hline 42 & Complejo Pampa Grande & $\mathrm{Si}$ & $\mathrm{Si}$ & & $x$ & \\
\hline 43 & La Puntilla & No & $\mathrm{Si}$ & & $x$ & \\
\hline 44 & Cerro Mulato & No & $\mathrm{Si}$ & $x$ & & \\
\hline 45 & Cerro Raca Rumi 1 & No & $\mathrm{Si}$ & $x$ & & \\
\hline 46 & Cerro Raca Rumi 2 & No & $\mathrm{Si}$ & $x$ & & \\
\hline 47 & Cerro Mal Paso & No & $\mathrm{Si}$ & $x$ & & \\
\hline 48 & Hacienda Cuculí & $\mathrm{Si}$ & No & $x$ & & \\
\hline 49 & Huaca Pais & $\mathrm{Si}$ & No & $x$ & & \\
\hline 50 & Huaca Magin & $\mathrm{Si}$ & No & $x$ & & \\
\hline 51 & San Juan de Tinajones & No & No & $x$ & & \\
\hline 52 & Quebrada Desaguadero & No & $\mathrm{Si}$ & $x$ & & \\
\hline 53 & Huaca Desaguadero & $\mathrm{Si}$ & No & $x$ & & \\
\hline 54 & Cerro Garraspiña & $\mathrm{Si}$ & No & & $x$ & \\
\hline
\end{tabular}




\begin{tabular}{|c|c|c|c|c|c|c|}
\hline \multirow{3}{*}{$\mathbf{N}^{\circ}$} & \multicolumn{3}{|c|}{ Sector cuenca hidrográfica Motupe - La Leche } & & & \\
\hline & \multirow{2}{*}{ Sitio Arqueológico } & \multirow{2}{*}{ Lluvias } & \multirow{2}{*}{$\begin{array}{c}\text { Derrumbes y } \\
\text { deslizamientos }\end{array}$} & \multicolumn{3}{|c|}{ Grado de afectación } \\
\hline & & & & Alta & Media & Baja \\
\hline 1 & Complejo Arqueológico La Pava 1 & $\mathrm{Si}$ & $\mathrm{Si}$ & & $x$ & \\
\hline 2 & Complejo Arqueológico La Pava 2 & $\mathrm{Si}$ & $\mathrm{Si}$ & & $x$ & \\
\hline 3 & Huaca Sandoval & $\mathrm{Si}$ & No & $x$ & & \\
\hline 4 & Huaca Paredones & $\mathrm{Si}$ & No & $x$ & & \\
\hline 5 & Huaca Julio García & $\mathrm{Si}$ & No & $x$ & & \\
\hline 6 & Huaca Santa María & $\mathrm{Si}$ & No & $\mathrm{x}$ & & \\
\hline 7 & Solecape & & & & & \\
\hline 8 & Huaca Los Perros & $\mathrm{Si}$ & $\mathrm{Si}$ & & $x$ & \\
\hline 9 & Huaca El Pueblo & $\mathrm{Si}$ & Si & & $x$ & \\
\hline 10 & Huaca San Genaro Chico & $\mathrm{Si}$ & No & $x$ & & \\
\hline 11 & Huaca Cruz de la Esperanza 1 & $\mathrm{Si}$ & No & $x$ & & \\
\hline 12 & Huaca Cruz de la Esperanza 2 & $\mathrm{Si}$ & No & $x$ & & \\
\hline 13 & Huaca Cruz de la Esperanza 3 & $\mathrm{Si}$ & No & $x$ & & \\
\hline 14 & Huaca Manuelon & $\mathrm{Si}$ & No & & $x$ & \\
\hline 15 & Huaca Túcume Viejo & $\mathrm{Si}$ & $\mathrm{Si}$ & $x$ & & \\
\hline 16 & Templo Colonial Túcume Viejo & $\mathrm{Si}$ & Si & & $x$ & \\
\hline 17 & Huaca El Sol & $\mathrm{Si}$ & $\mathrm{Si}$ & $x$ & & \\
\hline 18 & Huaca Larga & $\mathrm{Si}$ & $\mathrm{Si}$ & & $x$ & \\
\hline 19 & Complejo Arqueológico Huaca Bandera & $\mathrm{Si}$ & $\mathrm{Si}$ & & $x$ & \\
\hline 20 & Huaca Rodriguez & $\mathrm{Si}$ & No & $x$ & & \\
\hline 21 & Huaca Acosta & $\mathrm{Si}$ & No & $x$ & & \\
\hline 22 & Cerro Escute & $\mathrm{Si}$ & $\mathrm{Si}$ & $x$ & & \\
\hline 23 & Huaca Rivera & $\mathrm{Si}$ & No & $x$ & & \\
\hline 24 & Huaca Poma 3 & $\mathrm{Si}$ & No & $x$ & & \\
\hline 25 & Huaca Riendero 1 & $\mathrm{Si}$ & No & $x$ & & \\
\hline 26 & Huaca Riendero 2 & $\mathrm{Si}$ & No & $x$ & & \\
\hline 27 & Complejo Arqueológico Jotoro & $\mathrm{Si}$ & $\mathrm{Si}$ & & $x$ & \\
\hline 28 & Huaca Las Cardosas & $\mathrm{Si}$ & No & $x$ & & \\
\hline 29 & El Mirador & $\mathrm{Si}$ & No & $x$ & & \\
\hline 30 & El Marqués & $\mathrm{Si}$ & No & $\mathrm{x}$ & & \\
\hline 31 & Chililí & $\mathrm{Si}$ & $\mathrm{Si}$ & $x$ & & \\
\hline 32 & Complejo Arqueológico Mondragón & $\mathrm{Si}$ & $\mathrm{Si}$ & $x$ & & \\
\hline 33 & Huaca Palo Blanco & $\mathrm{Si}$ & $\mathrm{Si}$ & $x$ & & \\
\hline 34 & Huaca Palo Blanco 1 & $\mathrm{Si}$ & $\mathrm{Si}$ & $\mathrm{x}$ & & \\
\hline 35 & Huaca Las Juntas & $\mathrm{Si}$ & $\mathrm{Si}$ & $x$ & & \\
\hline 36 & Cerro Sonolipe & $\mathrm{Si}$ & $\mathrm{Si}$ & $x$ & & \\
\hline 37 & Complejo Arqueológico Apurlec & $\mathrm{Si}$ & $\mathrm{Si}$ & $x$ & & \\
\hline 38 & Huaca Colorada & $\mathrm{Si}$ & $\mathrm{Si}$ & $\mathrm{x}$ & & \\
\hline 39 & Cerro La Virgen & $\mathrm{Si}$ & $\mathrm{Si}$ & & $x$ & \\
\hline \multicolumn{7}{|c|}{ Fuente: Martínez, 2015.} \\
\hline
\end{tabular}


sos de deterioro en los elementos que deberían proteger. Esto se puede observar en los diferentes sitios arqueológicos que cuentan con coberturas de protección elaboradas en base a esteras con entramado de caña Guayaquil o en la técnica de cobertura tipo sándwich (estera, plástico, estera), que por la calidad deleznable de los materiales y frente a la exposición a la intemperie, principalmente, tienden a desintegrarse y depositarse sobre las estructuras que cobijan. En muchos casos ocasionan áreas cubiertas de material de desecho, por lo que se convierten en un medio para la proliferación de agentes biológicos en su interior, iniciando el proceso de deterioro de los elementos expuestos:

"por lo tanto es muy importante considerar la utilización de nuevos elementos de protección más livianos y con cualidades aislantes que actúen eficazmente frente el impacto de los agentes climáticos considerando las premisas de adaptación al contexto generando propuestas que dialoguen armoniosamente con estos elementos tan sensibles provenientes del pasado y que reclaman una correcta protección de lo que aún queda en pie". (World Monuments Fund, Fundación Pedro y Elena Hernándes A.C., Fomento Cultural Banamex, 2011, p. 19)

Las actividades de prevención se establecieron con la finalidad de intervenir en todas las estructuras arqueológicas vulnerables con la finalidad de "conservar la unidad paisajísti$\mathrm{ca}$ - cultural deteniendo los procesos de cambios irreversibles asegurando su conservación..." (Servicio Natural de Áreas Naturales Protegidas por el Estado, 2011, p. 29).

\section{Referencias}

Abad, C. (2015). Diagnóstico territorial para la elaboración del Plan Estratégico de Manejo de los Sitios Arqueológicos de Lambayeque: Elaboración, cartografía y SIG. Lima, Perú: Unidad Ejecutora 005 Naylamp Lambayeque.

Castañeda, J. et al. (2015). Templo virreinales de los valles de Lambayeque. Lima, Perú: Fondo Editorial de la Universidad de San Martín de Porres.

Coahila, A. (2015). Plan de acción inmediata ante la probable ocurrencia del fenómeno El Niño 2015- 2016. Chiclayo, Perú: Unidad Ejecutora 005 Naylamp Lambayeque.

Coahila, A. (2016). Informe de actividades de prevención por el fenómeno El Niño etapa 2015 2016. Chiclayo, Perú: Unidad Ejecutora 005 Naylamp Lambayeque.

Laínez, J. (2015). Elaboración del plan de seguridad local frente al fenómeno El Niño etapa 20152016. Chiclayo, Perú: Unidad Ejecutora 005 Naylamp Lambayeque.

Martínez, G. (2015). Diagnóstico territorial para la elaboración del Plan Estratégico de Manejo de los Sitios Arqueológicos de Lambayeque. Chiclayo, Perú: Unidad Ejecutora 005 Naylamp Lambayeque.

Morales, R. (2014a). Informe de evaluación y diagnostico físico - mecánico del patrimonio cultural edificado / costa norte del Perú (valles Tumbes a Casma). Trujillo, Perú: Programa de Prevención ENSO 2014.

Morales, R. (2014b). Informe final del estudio de las condiciones para el patrimonio cultural edificado en la costa norte del Perú (valles de Tumbes a Casma). Trujillo, Perú: Programa de Prevención ENSO 2014.

Servicio Natural de Áreas Naturales Protegidas por el Estado. (2011). Plan Maestro del Santuario Histórico Bosque de Pómac 2011-2016. Lima, Perú: Servicio Natural de Áreas Naturales Protegidas por el Estado.

World Monuments Fund, Fundación Pedro y Elena Hernándes A.C., Fomento Cultural Banamex. (2011). Cubiertas arquitectónicas en contextos arqueológicos. México DF: Fomento Cultural Banamex. 